\title{
The joint impact of bankruptcy costs, fire sales and cross-holdings on systemic risk in financial networks
}

\author{
Stefan Weber • Kerstin Weske
}

Received: 15 January 2017 / Accepted: 30 May 2017 / Published online: 26 June 2017

(c) The Author(s). 2017 Open Access This article is distributed under the terms of the Creative Commons Attribution 4.0 International License (http://creativecommons.org/licenses/by/4.0/), which permits unrestricted use, distribution, and reproduction in any medium, provided you give appropriate credit to the original author(s) and the source, provide a link to the Creative Commons license, and indicate if changes were made.

\begin{abstract}
The paper presents a comprehensive model of a banking system that integrates network effects, bankruptcy costs, fire sales, and cross-holdings. For the integrated financial market we prove the existence of a price-payment equilibrium and design an algorithm for the computation of the greatest and the least equilibrium. The number of defaults corresponding to the greatest price-payment equilibrium is analyzed in several comparative case studies. These illustrate the individual and joint impact of interbank liabilities, bankruptcy costs, fire sales and cross-holdings on systemic risk. We study policy implications and regulatory instruments, including central bank guarantees and quantitative easing, the significance of last wills of financial institutions, and capital requirements.
\end{abstract}

Keywords Systemic risk · Financial contagion · Financial network ·

Cross-holdings $\cdot$ Fire sales $\cdot$ Bankruptcy costs

JEL Classification G01 - G21 - D85.

\section{Introduction}

"Systemic risk refers to the risk that a financial system as a whole is susceptible to failures initiated by the characteristics of the system itself." 1 If strong links between financial institutions are present, a shock to only a small number

\footnotetext{
${ }^{1}$ See Feinstein et al. (2017)

Present Address:

S. Weber $(\square) \cdot$ K. Weske

Institut für Mathematische Stochastik, Leibniz Universität Hannover, Welfengarten 1, 30167

Hannover, Germany

e-mail: sweber@stochastik.uni-hannover.de
} 
of entities might propagate through the system and trigger substantial financial losses. Significant dependence can thus increase the risk of a system-wide breakdown.

Financial institutions influence each other via direct or indirect channels such as credit contracts, similar asset portfolios that are jointly exposed to price impact in market crises, and cross-shareholdings. Frictions like, e.g., bankruptcy costs may amplify the impact of the effect of the firms' interaction. The aim of the current paper consists in constructing and analyzing a comprehensive model that integrates all effects mentioned above. This multi-factor setting allows to assess regulatory policies in a robust manner. To the best of our knowledge, such a contribution is still missing in the literature.

(i) We prove the existence of a clearing equilibrium that is not necessarily unique and provide an algorithm for the computation of the greatest and the least equilibrium. The equilibrium is characterized by the vector of clearing payments and the price of the commonly held illiquid asset that is exposed to price effects.

(ii) We study the impact of bankruptcy costs, fire sales, and cross-holdings on systemic risk in numerical experiments. We demonstrate that fire sales and bankruptcy costs can trigger and amplify financial crises. Policies that mitigate their impact might significantly enhance the resilience of the financial system. Cross-holdings do, in contrast, have a stabilizing effect, if they can be exchanged for liquid assets. Central banks that engage in such a market can reduce the number of defaults in the system.

(iii) We study policy implications and regulatory instruments, including central bank guarantees, quantitative easing, the significance of last wills of financial institutions, and the efficiency of capital requirements. We find that capital adequacy ratios based on risk-weighted assets reduce systemic risk, if they are sufficiently high. However, they do not rely on any statistics that capture systemic risk in a proper way. Comparative statics show that capital adequacy ratios can be equal for varying parameters of our model that are associated with completely different levels of systemic risk. This demonstrates that classical capital adequacy ratios are a very rough instrument. A much better alternative are systemic risk measures that we analyze in the last section.

Previous papers do not allow an assessment of the robustness of their conclusions since they only focus on particular aspects of systemic risk neglecting all other driving factors. Our model, in contrast, shows to what extent causal relations that were previously discovered are preserved within a general framework; it also detects the differences that might occur. In summary, we find that many of our qualitative results are quite robust across different network structures and for a large number of driving factors. However, the relative importance of interacting contagion channels can only be characterized in the joint model. This justifies-for the first time from a general perspective - the relevance of previous approaches, but indicates at the same time that quantitative predictions and the design of regulatory policies require a more sophisticated analysis. 
Literature Our approach extends the equilibrium approach of Eisenberg and Noe (2001). Their seminal paper models interbank contagion within a network of nominal liabilities and proves the existence and uniqueness of a clearing payment vector that endogenously captures losses given default. At the same time, they construct an efficient algorithm for the computation of the clearing vector. Closely related empirical studies can, e.g., be found in Cont et al. (2013), Elsinger et al. (2006), Glasserman and Young (2015), and Upper (2011). These cast doubt that empirical patterns of contagious defaults can solely be explained by networks of nominal liabilities.

In this paper, we integrate multiple interaction channels and amplifying mechanisms of contagion, including bankruptcy costs, fire sales, and cross-holdings. While we investigate their joint impact, up to now the literature has only been studying these factors separately: Bankruptcy costs are, for example, considered by Elsinger (2009), Elliott et al. (2014), Rogers and Veraart (2013), and Glasserman and Young (2015); cross-holdings, e.g., by Elsinger (2009), Elliott et al. (2014), Fischer (2014), Suzuki (2002), and Karl and Fischer (2014). Cifuentes et al. (2005) incorporate fire sales into the setting of Eisenberg and Noe (2001); their approach is further extended by Amini et al. (2013), Chen et al. (2016), Gai and Kapadia (2010), Nier et al. (2007), and Feinstein (2017). Most of these papers consider only one extension of the basic framework. ${ }^{2}$ For a detailed review of the literature see also Staum (2013).

All of these mechanisms are important channels of contagion. In contrast to direct liabilities and cross-holdings that are described by network structures, fire sales are a global transmission mechanism. It is, for example, defined in Shleifer and Vishny (2011):

"A fire sale is essentially a forced sale of an asset at a dislocated price. The asset sale is forced in the sense that the seller cannot pay creditors without selling assets. The price is dislocated because the highest potential bidders are typically involved in a similar activity as the seller, and are therefore themselves indebted and cannot borrow more to buy the asset. Indeed, rather than bidding for the asset, they might be selling similar assets themselves. Assets are then bought by nonspecialists who, knowing that they have less expertise with the assets in question, are only willing to buy at valuations that are much lower."

Evidence is discussed in several papers including Brunnermeier (2009), Coval and Stafford (2007), Cont and Wagalath (2016), Jotikasthira et al. (2012), Khandani and Lo (2011), and Shleifer and Vishny (1992). In real markets, fire sales typically refer to the liquidation of portfolios. Empirical data show that this is related to increased correlations as well as price impact. A single representative illiquid asset can thus be used as a first approximation. This is the approach that we take in our model in order to keep the suggested framework simple.

\footnotetext{
${ }^{2}$ Elliott et al. (2014) and Elsinger (2009) consider both cross-holdings and bankruptcy costs. However, the underlying network model of Elliott et al. (2014) does not explicitly feature direct liabilities, but aggregates instead all dependencies linearly including cross-holdings. Elsinger (2009) uses a modified Eisenberg-Noe model, but only includes a stylized form of bankruptcy costs. This is primarily done in order to illustrate that the profitability of bailouts depends on these costs.
} 
Outline The paper is organized as follows. In the section "An integrated financial network model", we present our model of the financial system and provide a preliminary analysis of net worth, price impact, and clearing payment vectors. The existence of a price-payment equilibrium consisting of a clearing payment vector and a clearing price of the illiquid asset is demonstrated in the section "Existence of equilibria and an algorithm for their computation". Moreover, we provide an extension of the fictitious default algorithm of Eisenberg and Noe (2001) in order to compute the greatest and least equilibrium. The section "Case studies" focuses on numerical case studies which constitute a key part of our paper. These illustrate the individual and joint impact of bankruptcy costs, fire sales, and cross-holdings on systemic risk, measured as the number of defaults in the greatest price-payment equilibrium. We describe various regulatory policies and analyze their efficiency. The main conclusions and questions for future research are discussed in the section "Conclusion". All proofs of the results in the sections "An integrated financial network model" and "Existence of equilibria and an algorithm for their computation" are presented in the section "Proofs".

\section{An integrated financial network model}

We analyze default in a one-period interbank market model in which banks are connected to each other via three different channels:

- Direct liabilities: Banks have nominal liabilities against each other. These liabilities are promises that will only partially be fulfilled if some of the banks default.

- Fire sales: If the portfolios of different banks include common assets, changes in asset prices simultaneously influence the net worths of these banks. Common holdings may give rise to substantial systemic risk, if illiquid assets are sold in large quantities and prices decrease significantly. For simplicity, our model assumes the existence of a single (representative) illiquid asset.

- Cross-holdings: Banks may, in addition, hold shares of each other. In this case, the net worths of banks depends on the net worths of other banks due to these cross-holdings.

The single period is interpreted as a snapshot of a banking system that continues to exist afterwards. The net worth of each bank in the financial network depends on the realized payments, the price of the commonly held illiquid asset, and the net worths of the other banks. In the first step, we will describe how the value of asset holdings of an individual bank can be computed if these three key factors are exogenously fixed. In the second step, we will construct and analyze an equilibrium model that allows an endogenous computation of the net worths of all banks, a clearing payment vector, and a realized average price of the illiquid asset.

\section{Assets and liabilities}

Letting $\mathcal{N}=\{1, \ldots, n\}$ be the set of banks in the financial system, we denote by $p \in \mathbb{R}_{+}^{n}$ the realized payments of the banks, by $w \in \mathbb{R}_{+}^{n}$ the vector of net worths of the banks, and by $q \in \mathbb{R}_{+}$the price of the representative 
illiquid asset. In the first step, we suppose that these quantities are exogenously specified.

External Assets As suggested by Cifuentes et al. (2005), we consider banks that hold two assets which are external to the banking system: an amount of $r \in \mathbb{R}_{+}^{n}$ shares of a liquid asset (e.g., cash) and $s \in \mathbb{R}_{+}^{n}$ shares of an illiquid asset. Assuming that the liquid asset's price remains constant at one monetary unit, the value of bank $i$ 's external assets is given by $r_{i}+s_{i} q$, if the price of the illiquid asset is $q$.

Liabilities Each bank has nominal liabilities to the other banks for the considered time horizon. Analogous to Eisenberg and Noe (2001), we suppose that these liabilities are represented by a nominal liabilities matrix $L \in \mathbb{R}^{n \times n}$ : for all $i, j \in \mathcal{N}$, $L_{i j} \geq 0$ describes the nominal obligation of bank $i$ towards bank $j$; no bank may hold a liability against itself, i.e., $L_{i i}=0$ for all $i \in \mathcal{N}$. In addition, banks may have further liabilities $l \in \mathbb{R}_{+}^{n}$ to entities outside the banking system; here, the component $l_{i}$ is interpreted as the liability of bank $i$ to the outside.

The vector of total liabilities $\bar{p}$ captures all liabilities of the banks in the system; i.e., its component $\bar{p}_{i}$ equals the total liabilities of bank $i$ and is given by $\bar{p}_{i}=\sum_{j \in \mathcal{N}} L_{i j}+l_{i}$, for $i \in \mathcal{N}$. If all banks are able to fulfill their total obligations, $\bar{p}$ indeed equals the realized payments $p$ of the banks. If, in contrast, some banks do not possess sufficient resources to meet their obligations, then $p \leq \bar{p}$, where the inequality is interpreted componentwise.

Following Eisenberg and Noe (2001), we assume that in case of bank $i$ 's default, its realized payments $p_{i}<\bar{p}_{i}$ will be distributed proportionally among its creditors according to the size of each creditor's claim. Therefore, we define the relative liabilities matrix $\Pi \in \mathbb{R}^{n \times n}$ by $\Pi_{i j}=L_{i j} / \bar{p}_{i}$, if $\bar{p}_{i}>0$, and $\Pi_{i j}=0$, otherwise. Thus, the entry $\Pi_{i j}$ captures the size of the interbank obligations of bank $i$ towards bank $j$ in proportion to the size of $i$ 's total liabilities. This implies that for a given vector of realized payments $p$, the value of bank $i$ 's interbank claims is given by $\sum_{j \in \mathcal{N}} \Pi_{j i} p_{j}$.

Cross-Holdings Each bank may hold shares of the other banks. Following Elsinger (2009), these holdings will be captured by a cross-holdings matrix $C \in \mathbb{R}^{n \times n}$ : the component $C_{i j}$ denotes the fraction of bank $i$ 's equity that is held by bank $j$. We assume that the cross-holdings are nonnegative, i.e., $C_{i j} \geq 0$ for all $i, j \in \mathcal{N}$, and that a bank is not allowed to hold shares of itself, i.e., $C_{i i}=0$ for all $i \in \mathcal{N}$. The technical assumption $\sum_{j \in \mathcal{N}} C_{i j}<1, i \in \mathcal{N}$, guarantees that the net worth of each bank, as introduced below, is well-defined. If both a cross-holdings matrix $C$ and a vector of positive net worths $w$ are given, the contribution of bank $i$ 's cross-holdings to its net worth is equal to $\sum_{j \in \mathcal{N}} C_{j i} w_{j}$. We also suppose limited liability of crossholdings, i.e., if bank $j$ 's net worth $w_{j}$ is negative, cross-holdings of bank $j$ do not negatively affect the net worths of other banks.

It is well-known that cross-holdings inflate the value of the financial system, see Brioschi et al. (1989), Fedenia et al. (1994), and Elliott et al. (2014). This, in particular, refers to the fact that the aggregated net worth of all banks will be larger than the value of total assets if cross-holdings are present. As argued in Brioschi et al. (1989), Fedenia et al. (1994), and Elliott et al. (2014), net worths need to be adjusted by an 
auxiliary factor that guarantees the conservation of value in the system. The market value of bank $i$ should thus be computed as $\left(1-\sum_{j \in \mathcal{N}} C_{i j}\right) w_{i}$ for $w_{i} \geq 0$.

Total net worth We will now describe how each bank's net worth is calculated. In order to fulfill its obligation $\bar{p}_{i}$, bank $i$ will first use its liquid external assets $r_{i}$ and its interbank revenues $\sum_{j \in \mathcal{N}} \Pi_{j i} p_{j}$. If these are insufficient, the bank is left with its illiquid asset and cross-holdings. We assume that bank $i$ 's cross-holdings $\sum_{j \in \mathcal{N}} C_{j i} w_{j}$ can be exchanged against cash (possibly involving central banks or governments). However, we suppose that bank $i$ can only realize a fraction of $\lambda_{i} \in$ $[0,1]$. An alternative way to model price impact of cross-holdings liquidation via inverse demand functions is presented in "Appendix 2. Cross-holdings with price impact".

Each bank decides on the order of liquidation. This decision is captured by an indicator variable $\mathbb{I}_{i} \in\{0,1\}$, where $\mathbb{I}_{i}=1$ represents the case that bank $i$ exchanges its total cross-holdings against cash before it starts selling the illiquid asset; $\mathbb{I}_{i}=0$ refers to the reversed order of liquidation. Banks liquidate their cross-holdings proportionally, i.e., the percentage of cross-holdings that are exchanged against cash can be computed as

$$
\nu_{i}(p, q, w):=\min \left(\frac{\max \left(\bar{p}_{i}-r_{i}-\sum_{j \in \mathcal{N}} \Pi_{j i} p_{j}-\left(1-\mathbb{I}_{i}\right) s_{i} q, 0\right)}{\lambda_{i} \sum_{j \in \mathcal{N}} C_{j i} \max \left(w_{j}, 0\right)}, 1\right) \in[0,1] .
$$

The remaining share $1-v_{i}(p, q, w)$ remains on the bank's balance sheet and is not subject to the price impact modeled by the factor $\lambda_{i}$. Setting $\mu_{i}(p, q, w):=$ $v_{i}(p, q, w) \lambda_{i}+1-v_{i}(p, q, w), i \in \mathcal{N}$, the net worth of bank $i$ is given by

$$
w_{i}=r_{i}+s_{i} q+\sum_{j \in \mathcal{N}} \Pi_{j i} p_{j}+\mu_{i}(p, q, w) \sum_{j \in \mathcal{N}} C_{j i} \max \left(w_{j}, 0\right)-\bar{p}_{i} .
$$

The bank is in default if it cannot cover its liabilities, i.e., if $w_{i}<0$.

As mentioned before, due to cross-holdings, the net worths of the banks differ from their market values, see Brioschi et al. (1989), Fedenia et al. (1994), and Elliott et al. (2014). We will, however, focus on default count statistics which can be computed in terms of the vector of net worths. Market values will only be considered explicitly in the numerical case studies.

As defined above, the net worth of bank $i$ depends on the realized payments $p$, the price $q$ of the illiquid asset, and all other banks' net worths $w$. In the following sections, we provide a method to derive these three key quantities endogenously.

\section{Net worth}

Suppose first that $p$ and $q$ are fixed. In this situation, our aim is to define an equilibrium vector of the net worths of the banks. To simplify the notation, we write $\mathbf{0}:=$ $(0, \ldots, 0)^{T}, \mathbf{1}:=(1, \ldots, 1)^{T} \in \mathbb{R}^{n}$, set $a \vee b:=\left(\max \left(a_{1}, b_{1}\right), \ldots, \max \left(a_{n}, b_{n}\right)\right)$ for $a, b \in \mathbb{R}^{n}$ and denote by $\operatorname{diag}(\mu(p, q, w))$ the diagonal matrix whose diagonal entries are given by the vector $\mu(p, q, w):=\left(\mu_{1}(p, q, w), \ldots, \mu_{n}(p, q, w)\right)^{T}$. 
Definition 2.1 For $p \in \mathbb{R}_{+}^{n}, q \in \mathbb{R}_{+}$, an essential net worths vector is a fixedpoint vector $w^{*}(p, q) \in \mathbb{R}^{n}$ such that

$$
w^{*}(p, q)=\Psi\left(w^{*}(p, q)\right)
$$

where the function $\Psi: \mathbb{R}^{n} \rightarrow \mathbb{R}^{n}$ is defined by

$$
\Psi(w):=r+s q+\Pi^{T} p+\operatorname{diag}(\mu(p, q, w)) C^{T}(w \vee \mathbf{0})-\bar{p} .
$$

The essential net worths vector is defined as a solution of the non-linear fixedpoint problem (1). The following lemma shows that this equation always possesses a unique solution. A proof of this result is given in the section "Proofs".

Lemma 2.2 For all $p \in \mathbb{R}_{+}^{n}, q \in \mathbb{R}_{+}$:

(a) The essential net worths vector $w^{*}(p, q)$ exists and is unique.

(b) The essential net worths vector is increasing in payments and prices:

$$
\begin{array}{ll}
p^{1} \geq p^{2} & \Longrightarrow \quad w^{*}\left(p^{1}, q\right) \geq w^{*}\left(p^{2}, q\right), \\
q^{1} \geq q^{2} & \Longrightarrow \quad w^{*}\left(p, q^{1}\right) \geq w^{*}\left(p, q^{2}\right) .
\end{array}
$$

\section{Price of the illiquid asset}

We will now explain how the clearing vector $p$ and the price $q$ of the illiquid asset can endogenously be derived. The presence of the illiquid asset enables us to incorporate the effect of fire sales into the model. As described in the introduction, the basic economic idea is that, if a bank is unable to pay its outstanding debt in the considered period using its shares of the liquid asset and interbank payments, it can sell a proportion of its illiquid asset holdings at the current market price. This triggers an increase in the supply of the asset that can decrease its market price during times of crisis. Consequently, other banks holding the same asset are also affected by such a price decline. In particular, if they need to sell an amount of the illiquid asset themselves, the proceeds from this transaction are diminished. At the same time, the price is further pushed down.

To integrate this idea into our model, we assume that there exists an exogenously given positive continuous inverse demand function for the illiquid asset $f:\left[0, \sum_{i \in \mathcal{N}} s_{i}\right] \rightarrow(0, \infty)$, such that the price $q$ of one unit of the illiquid asset is given by $q=f(\theta)$, where $\theta$ denotes the quantity of the illiquid asset that is sold in the market. We assume that $f(0)=q_{0}$ and that $\theta \mapsto f(\theta)$ is monotonically decreasing which indicates that the illiquid asset's price is decreasing in its supply. A fixed-point problem is present, since the amount sold depends on the price of the illiquid asset itself:

$$
q=f(\theta(p, q))
$$


for a given payment vector $p$ and where

$$
\theta(p, q):=\sum_{i \in \mathcal{N}} \min \left(\frac{\max \left[\bar{p}_{i}-r_{i}-\sum_{j \in \mathcal{N}} \Pi_{j i} p_{j}-\mathbb{I}_{i} \lambda_{i} \sum_{j \in \mathcal{N}} C_{j i} \max \left(w_{j}^{*}(p, q), 0\right), 0\right]}{q}, s_{i}\right)
$$

signifies the total amount of the illiquid asset that is sold. If $\mathbb{I}_{i}=1$, bank $i$ exchanges its total cross-holdings against cash before selling the illiquid asset and, obviously, this decreases the asset's supply $\theta$; here, $w^{*}(p, q)$ denotes the unique vector of essential net worths as introduced in Definition 2.1. We assume that all illiquid asset holdings are marked-to-market at the resulting price. Note that due to our assumptions, the price of the illiquid asset is both bounded from above by $q_{0}$ and from below by $q_{\text {min }}:=f\left(\sum_{i \in \mathcal{N}} s_{i}\right)$, since banks may at most sell their total holdings of the illiquid asset $\sum_{i \in \mathcal{N}} s_{i}$.

\section{Payment vector}

In the final step, we define a price-payment equilibrium that endogenously derives the price of the illiquid asset as well as a corresponding clearing vector. We integrate one more effect that influences the clearing process, namely bankruptcy costs. In reality, a fraction of the recovery value of the assets will be lost to the obligors in case of default due to, e.g., legal and administrative expenses. Observe that bankruptcy costs and fire sales impact systemic risk differently. Firstly, bankruptcy costs are only incurred in the case of a default, while fire sales may also occur if there are no defaults in the system. Secondly, a fire sale may affect banks that are not connected to the triggering bank via direct credit contracts. Fire sales are a global channel of contagion, while bankruptcy costs are an amplifier of credit risk.

Following Rogers and Veraart (2013), we introduce two new parameters $0 \leq \alpha \leq$ 1 and $0 \leq \beta \leq 1$, such that $1-\alpha$ and $1-\beta$ determine the frictional costs due to bankruptcy: A defaulting bank will only realize a fraction $\alpha$ of its external asset value, i.e., the value of the liquid and illiquid asset, and a fraction $\beta$ of its interbank asset value, i.e., the value of interbank claims and cross-holdings. We further postulate that the clearing process satisfies the criteria of proportionality, limited liability, and absolute priority of debt, as outlined by Eisenberg and Noe (2001). Finally, we define a price-payment equilibrium as follows.

Definition 2.3 A price-payment equilibrium is a pair $\left(p^{*}, q^{*}\right) \in[\mathbf{0}, \bar{p}] \times$ $\left[q_{\min }, q_{0}\right] \subseteq \mathbb{R}^{n+1}$, consisting of a clearing payment vector $p^{*}$ and a clearing price $q^{*}$, such that

$$
\left(p^{*}, q^{*}\right)=\Phi\left(p^{*}, q^{*}\right)
$$

where $\Phi: \mathbb{R}^{n+1} \rightarrow \mathbb{R}^{n+1}$ is the function defined by

$$
\Phi_{i}(p, q):= \begin{cases}\chi_{i}(p, q), & \text { for } i=1, \ldots, n \\ f(\theta(p, q)), & \text { for } i=n+1\end{cases}
$$


with

$$
\begin{aligned}
\chi_{i}(p, q) & := \begin{cases}\bar{p}_{i}, \\
\alpha\left[r_{i}+s_{i} q\right]+\beta\left[\eta_{i}(p, q)\right], & \text { if } r_{i}+s_{i} q+\eta_{i}(p, q) \geq \bar{p}_{i},\end{cases} \\
\eta_{i}(p, q) & :=\sum_{j \in \mathcal{N}} \Pi_{j i} p_{j}+\mu_{i}(p, q) \sum_{j \in \mathcal{N}} C_{j i} \max \left(w_{j}^{*}(p, q), 0\right), \\
\mu_{i}(p, q) & =v_{i}(p, q) \lambda_{i}+1-v_{i}(p, q), \\
v_{i}(p, q) & =\min \left(\frac{\max \left(\bar{p}_{i}-r_{i}-\sum_{j \in \mathcal{N}} \Pi_{j i} p_{j}-\left(1-\mathbb{I}_{i}\right) s_{i} q, 0\right)}{\lambda_{i} \sum_{j \in \mathcal{N}} C_{j i} \max \left(w_{j}^{*}(p, q), 0\right)}, 1\right),
\end{aligned}
$$

and

$$
\theta(p, q):=\sum_{i \in \mathcal{N}} \min \left(\frac{\max \left(\bar{p}_{i}-r_{i}-\sum_{j \in \mathcal{N}} \Pi_{j i} p_{j}-\mathbb{I}_{i} \lambda_{i} \sum_{j \in \mathcal{N}} C_{j i} \max \left(w_{j}^{*}(p, q), 0\right), 0\right)}{q}, s_{i}\right) .
$$

In the combined equilibrium, the banks' clearing payments $p^{*}$ are given as the fixed point of the function $\chi$, and a clearing price of the illiquid asset $q^{*}$ is found as a fixed point of the inverse demand function $f$. Hence, bank $i$ pays its total liabilities $\bar{p}_{i}$, if its total asset value consisting of its external asset value $r_{i}+s_{i} q^{*}$ and interbank asset value $\eta_{i}\left(p^{*}, q^{*}\right)$ exceeds its liabilities. If this is not the case, bank $i$ is in default and receives (and due to absolute priority of debt also pays out) only the given fractions $\alpha$ and $\beta$ of its external and interbank asset value, respectively. The interbank asset value of bank $i, \eta_{i}\left(p^{*}, q^{*}\right)$, depends on the proportion of cross-holdings that are exchanged against liquid assets, $v_{i}\left(p^{*}, q^{*}\right)$, and this proportion is multiplied by $\lambda_{i} \in$ $[0,1]$ as defined in $\mu_{i}\left(p^{*}, q^{*}\right)$. Finally, the amount of the illiquid asset that is sold in equilibrium is given by $\theta\left(p^{*}, q^{*}\right)$.

The price-payment equilibrium provides a solution to an integrated financial system which is characterized by $(\Pi, \bar{p}, r, s, \alpha, \beta, \lambda, f, C, \mathbb{I})$; here, $\lambda=\left(\lambda_{1}, \ldots, \lambda_{n}\right) \in$ $[0,1]^{n}$ and $\mathbb{I}=\left(\mathbb{I}_{1}, \ldots, \mathbb{I}_{n}\right) \in\{0,1\}^{n}$. It admits a joint analysis of a network of liabilities, bankruptcy costs, cross-holdings, and fire sales as well as an analysis of models that incorporate only some of these effects. Namely, by choosing $\alpha=\beta=1, s=\mathbf{0}$, or $C$ as the zero $n \times n$ matrix, we can simply exclude the corresponding extensions from our system. This shows that the models of, e.g., Eisenberg and Noe (2001), Rogers and Veraart (2013), Cifuentes et al. (2005), and Elsinger (2009) are special cases of our integrated financial system.

\section{Existence of equilibria and an algorithm for their computation}

The current section analyzes the existence and computation of equilibria. All proofs are provided in the section "Proofs". We consider the ordered vector space $\left\langle\mathbb{R}^{n+1}, \leq\right\rangle$ equipped with the partial order $x \leq y: \Leftrightarrow x_{i} \leq y_{i} \forall i=1, \ldots, n+1$ and use the notation $x<y: \Leftrightarrow\left(x \leq y\right.$ and $\left.\exists i: x_{i} \neq y_{i}\right)$. We will also use this ordering on linear subspaces. The following lemma states elementary properties of the function $\Phi$, see Definition 2.3. 
Lemma 3.1 The function $\Phi$ has the following properties:

(a) $\Phi$ is bounded: For all $(p, q) \in[\mathbf{0}, \bar{p}] \times\left[q_{\min }, q_{0}\right]: \Phi(p, q) \geq\left(\mathbf{0}, q_{\min }\right)$ and $\Phi(p, q) \leq\left(\bar{p}, q_{0}\right)$

(b) $\Phi$ is monotone: For $\left(p^{1}, q^{1}\right) \leq\left(p^{2}, q^{2}\right)$ : $\Phi\left(p^{1}, q^{1}\right) \leq \Phi\left(p^{2}, q^{2}\right)$.

Lemma 3.1 enables us to apply Tarski's fixed-point theorem (Tarski (1955, Theorem 1)) to the function $\Phi$ proving the existence of equilibria.

Theorem 3.2 Let $(\Pi, \bar{p}, r, s, \alpha, \beta, \lambda, C, \mathbb{I})$ be an integrated financial system. Then, there always exist a unique greatest and least price-payment equilibrium $\left(p^{+}, q^{+}\right)$and $\left(p^{-}, q^{-}\right)$, i.e., for every price-payment equilibrium $\left(p^{*}, q^{*}\right)$ it holds that

$$
\left(p^{-}, q^{-}\right) \leq\left(p^{*}, q^{*}\right) \leq\left(p^{+}, q^{+}\right) \text {. }
$$

Remark 3.3 While the clearing payment vector is unique within the basic setting of Eisenberg and Noe (2001) under certain technical conditions, extensions allowing separately for bankruptcy costs or fire sales may lead to multiple clearing vectors, see Rogers and Veraart (2013, Example 3.3) and Chen et al. (2016, Example 7). This observation applies, in particular, to our integrated financial system as shown by an example in "Appendix 1. Example: price-payment equilibria". The example also demonstrates that the set of equilibria is not necessarily connected.

Amini et al. (2016) prove uniqueness of price-payment equilibria in a model with fire sales under the additional condition on the inverse demand function $f$ that $x \mapsto x f(x)$ is strictly increasing. They provide the following rational for their assumption: If there exists a subinterval $I:=\left(x_{0}, x_{1}\right)$ with $I \quad \ni \quad x \mapsto$ $x f(x)$ decreasing, rational banks would never choose to sell a suboptimal amount $x \in I$ of the illiquid asset; they would instead liquidate less, i.e., only the quantity $x_{0}$.

The argument of Amini et al. (2016) relies on implicit assumptions. First, it requires that banks understand both the mechanism of price formation as well as their own price impact. Second, the price of the illiquid asset depends on the total quantity that is sold which would have to be known; it does not only depend on the amount that is sold by an individual bank. However, in contrast to the price, the total quantity sold is hardly observable in reality. Third, Amini et al. (2016) interpret the one-stage model literally; the latter could also be seen as a simplified static picture of the true dynamic processes of prices sliding down the slide while banks continue to liquidate their holdings over time. For simplicity, we decided to model banks as price takers and do not impose the additional condition of Amini et al. (2016).

We now explain how equilibria can be determined, generalizing the fictitious default algorithm of Eisenberg and Noe (2001), and the procedures of Rogers and Veraart (2013), and Amini et al. (2013). Our method allows the computation of the greatest and least price-payment equilibrium, see Algorithm 3.4 and Remark 3.6 below. 
Algorithm 3.4 Set $k=0,\left(p^{(0)}, q^{(0)}\right):=\left(\bar{p}, q_{0}\right), \mathcal{D}_{-1}:=\varnothing$ and determine the starting essential net worths vector $w^{(0)}:=w^{*}\left(p^{(0)}, q^{(0)}\right)$ using fixed-point iteration. Determine the sets of initially defaulting and surviving banks

$$
\mathcal{D}_{0}:=\left\{i \in \mathcal{N} \mid w_{i}^{(0)}<0\right\} \quad \text { and } \quad \mathcal{S}_{0}:=\left\{i \in \mathcal{N} \mid w_{i}^{(0)} \geq 0\right\} .
$$

If $\mathcal{D}_{0}=\mathcal{D}_{-1}$ and no bank has to liquidate its illiquid asset holdings, i.e., for all $i \in \mathcal{N}$ :

$$
r_{i}+\sum_{j \in \mathcal{N}} \Pi_{j i} p_{j}^{(0)}+\mathbb{I}_{i} \lambda_{i} \sum_{j \in \mathcal{N}} C_{j i} \max \left(w_{j}^{(0)}, 0\right) \geq \bar{p}_{i},
$$

terminate. Otherwise, go to Step 2.

Step 1: Determine the sets of defaulting and surviving banks

$$
\mathcal{D}_{k}:=\left\{i \in \mathcal{N} \mid w_{i}^{(k)}<0\right\} \quad \text { and } \quad \mathcal{S}_{k}:=\left\{i \in \mathcal{N} \mid w_{i}^{(k)} \geq 0\right\} .
$$

If $\mathcal{D}_{k}=\mathcal{D}_{k-1}$, terminate. Otherwise, go to Step 2 .

Step 2: $\quad$ Set $p_{i}^{(k+1)}=\bar{p}_{i}$ for all $i \in \mathcal{S}_{k}, p_{i}^{(k+1)}=x_{i}$ for all $i \in \mathcal{D}_{k}, q^{(k+1)}=y$, and $w^{(k+1)}=w^{*}(x, y)$, where $(x, y)$ is determined as the maximal solution to the following system of equations:

$$
\begin{gathered}
x_{i}=\alpha\left[r_{i}+s_{i} y\right]+\beta\left[\sum_{j \in \mathcal{D}_{k}} \Pi_{j i} x_{j}+\sum_{j \in \mathcal{S}_{k}}\left[\Pi_{j i} \bar{p}_{j}+\lambda_{i} C_{j i} \max \left(w_{j}^{*}(x, y), 0\right)\right], \quad \forall i \in \mathcal{D}_{k}, \quad\right. \text { (4) } \\
y=f\left(\sum_{i \in \mathcal{D}_{k}} s_{i}+\sum_{i \in \mathcal{S}_{k}} \min \left(\frac{\zeta_{i}^{(k)}(x, y)}{y}, s_{i}\right)\right), \\
\zeta_{i}^{(k)}(x, y):=\max \left(\bar{p}_{i}-r_{i}-\sum_{j \in \mathcal{D}_{k}} \Pi_{j i} x_{j}-\sum_{j \in \mathcal{S}_{k}}\left[\Pi_{j i} \bar{p}_{j}+\mathbb{I}_{i} \lambda_{i} C_{j i} \max \left(w_{j}^{*}(x, y), 0\right)\right], 0\right), \quad \forall i \in \mathcal{S}_{k} .
\end{gathered}
$$

The (sloppy) notation $w^{*}(x, y)$ refers to the essential net worths vector corresponding to the payment vector $p^{(k+1)}$; its components are equal to $x_{i}$ for the defaulting banks $i \in \mathcal{D}_{k}$ and equal to $\bar{p}_{i}$ for the surviving banks $i \in \mathcal{S}_{k}$.

Set $k \rightarrow k+1$ and go to Step 1 .

The algorithm works as follows. Starting with the total liabilities vector for the payments and the initial price of the illiquid asset $q_{0}$, it calculates the set of those banks that will default even if all other banks pay their liabilities in full. This is the set of initially defaulting banks. If there are no initially defaulting banks and, in addition, no bank has to liquidate parts of its illiquid asset holdings, we immediately arrive at the end of the clearing process and terminate. Note that leaving out the extra condition (3) in the initial step may lead to an incorrect result if the contagion cascade is solely triggered by the asset price effect. This is due to the fact that the price of the illiquid asset and the corresponding payments must be adjusted if there are banks forced to sell the illiquid asset. The adjustment is made in Step 2 by solving the fixed-point Eqs. (4) and (5) simultaneously. Using the adjusted price-payment pair, in Step 1 of the next round, we calculate the set of defaulting banks that corresponds to it. If this default set equals the default set from the previous round, the algorithm 
terminates with the current pair of payments and price of the illiquid asset. Otherwise, the procedure continues until the set of defaulting banks does not change anymore.

The following theorem extends Rogers and Veraart (2013, Theorem 3.7) to the case of cross-holdings and fire sales.

Theorem 3.5 Algorithm 3.4 produces a sequence of price-payment pairs $\left(p^{(k)}, q^{(k)}\right)$ that decreases to the greatest price-payment equilibrium in at most $n+1$ iterations.

Remark 3.6 Algorithm 3.4 computes the least price-payment equilibrium, if we make the following changes:

- In the initial step: Set $\left(p^{(0)}, q^{(0)}\right)=\left(\mathbf{0}, q_{\min }\right), \mathcal{D}_{-1}=\mathcal{N}$ and terminate the algorithm if $\mathcal{D}_{0}=\mathcal{D}_{-1}$, i.e., condition (3) can be eliminated.

- In Step 2: Compute the minimal solution to the system of equations.

The iterations of the procedure that computes the least price-payment equilibrium can be viewed ${ }^{3}$ as a process in which financial health spreads throughout a system that is initially in default. The iterations of the algorithm that determines the greatest price-payment equilibrium describe, in contrast, how defaults spread in a system that initially is completely healthy. As we expect the latter situation to be more likely in real world financial markets, we focus on the greatest equilibrium when conducting our numerical case studies.

The greatest price-payment equilibrium $\left(p^{+}, q^{+}\right)$corresponds to a set of defaulting banks

$$
\mathcal{D}\left(p^{+}, q^{+}\right):=\left\{i \in \mathcal{N} \mid w_{i}^{*}\left(p^{+}, q^{+}\right)<0\right\}=\left\{i \in \mathcal{N} \mid p_{i}^{+}<\bar{p}_{i}\right\}
$$

that is directly provided by Algorithm 3.4. The cardinality of this set is a simple measure of systemic risk. In the following section, we will investigate how this quantity is affected by bankruptcy costs, cross-holdings, and fire sales—separately and jointly.

\section{Case studies}

The integrated financial system is characterized by a 10-tuple, ( $\Pi, \bar{p}, r, s, \alpha, \beta, \lambda$, $f, C, \mathbb{I}$ ). The relative liabilities matrix $\Pi$ and the cross-holdings matrix $C$ will be modeled as random quantities. In contrast to a deterministic approach, a probabilistic mechanism allows for an identification of the structure of a class of networks on the basis of appropriate statistical quantities. We choose two settings: Erdös-Rényi random networks (Erdös and Rényi (1959)), and a two-tiered (core-periphery) random graph model adapted to German interbank market data (extracted from Craig and von Peter (2014)). We also analyze an extension to multi-layer networks that capture heterogeneous business models.

\footnotetext{
${ }^{3}$ This interpretation is due to Rogers and Veraart (2013).
} 


\section{Erdös-Rényi random networks}

\section{Simulation methodology}

We use a simulation procedure similar to Elliott et al. (2014). Specifically, we choose two parameters $c_{\Pi} \in[0,1]$ and $d_{\Pi} \in[0, n-1]$ describing the level of integration and diversification of the relative liabilities network. The parameter $c_{\Pi}$ refers to the proportion of total liabilities that are spread across the interbank market while $d_{\Pi}$ describes the expected number of creditors of a bank therein. We generate a homogeneous weighted random network for $\Pi$ with $n$ nodes as follows:

1. Construct an adjacency matrix $A \in \mathbb{R}^{n \times n}$ by letting $A_{i j}, i \neq j \in \mathcal{N}$, be i.i.d. Bernoulli random variables, taking the value 1 with probability $d_{\Pi} /(n-1)$ and 0 with probability $1-d_{\Pi} /(n-1)$. Set $A_{i i}=0$ for all $i \in \mathcal{N}$.

2. For all banks $i \in \mathcal{N}$, set $d_{i}^{\text {out }}=\sum_{j \in \mathcal{N}} A_{i j}$, and let $\Pi_{i j}=c_{\Pi} / d_{i}^{\text {out }}$ if $A_{i j}=1$, otherwise $\Pi_{i j}=0, j \in \mathcal{N}$.

The parameter $d_{\Pi}$ is the average out-degree of the corresponding directed network, the parameter $c_{\Pi}$ is the row sum of the matrix $A$.

The cross-holdings matrix $C$ describes an interbank network as well and can be modeled according to an analogous mechanism. We denote by $c \in[0,1)$ the corresponding level of integration, and by $d \in[0, n-1]$ the level of diversification of the cross-holdings matrix. The parameter $c$ refers to the fraction of net worth that banks sell as cross-holdings to other banks, $d$ describes the expected number of shareholders within the interbank market. We assume that banks can liquidate crossholdings at their market value, possibly reduced to a fraction $\kappa \in[0,1)$. We thus set $\lambda_{i}=(1-c) \kappa, i \in \mathcal{N}$. Buyers could either be market participants or a central bank that tries to stabilize the financial system.

The number of shares $r$ of the liquid asset and the number of shares $s$ of the illiquid asset are specified in terms of two parameters $\delta$ and $\rho ; \delta$ denotes the size of a capital buffer, and $\rho$ the proportion of the illiquid asset:

1. Compute the random vector of the minimal value of assets that prevent the banks from defaulting (not considering cross-holdings): $h:=\left(\bar{p}-\Pi^{T} \bar{p}\right) \vee \mathbf{0}$.

2. Given a capital buffer $\delta>0$, set the overall external assets to be $e:=(1+\delta) h$. 3. Given a proportion $\rho \in[0,1]$ of the illiquid asset, let $r=(1-\rho) e$ and $s=\rho e$.

For simplicity, we use the parametric exponential inverse demand function $f(x)=$ $\exp (-\gamma x)$; alternative inverse demand functions can also be implemented within our framework. We assume that all banks follow the same rule regarding the order of liquidation. This means that either all banks exchange their total cross-holdings against cash before selling the illiquid asset (i.e., $\mathbb{I}=\mathbf{1}$ ), or that all banks first liquidate their total holdings of the illiquid asset before using cross-holdings (i.e., $\mathbb{I}=\mathbf{0}$ ).

Setting $n=100$ and $\bar{p}=\mathbf{1}$, the following parameters govern the simulation model:

$$
\left(c_{\Pi}, d_{\Pi}, \delta, \alpha, \beta, \rho, \gamma, c, d, \kappa, \mathbb{I}\right)
$$


Table 1 Extension parameters

\begin{tabular}{lll}
\hline Parameter & Description & Range of variation \\
\hline$\alpha$ & Realized fraction of external asset value in case of bankruptcy & {$[0.5,1]$} \\
$\beta$ & Realized fraction of interbank asset value in case of bankruptcy & {$[0,1]$} \\
$\rho$ & Proportion of the illiquid asset & {$[0,0.05]$} \\
$\gamma$ & Exponent of the inverse demand function & {$[0,1]$} \\
$c$ & Integration of the cross-holdings matrix & {$[0,0.9]$} \\
$d$ & Diversification of the cross-holdings matrix & {$[1,90]$} \\
$\kappa$ & Realized fraction of cross-holdings' market value & {$[0,1]$} \\
$\mathbb{I}$ & Order of liquidation & $\{\mathbf{0 , 1}\}$ \\
\hline
\end{tabular}

We fix $c_{\Pi}=0.15, d_{\Pi}=10$, and $\delta=0.01$, and vary the other parameters as indicated in Table 1.4

Methodological Remark 4.1 The numerical case studies are conducted as follows: $\Pi$ and $C$ are randomly sampled; the derived random quantities $r$ and $s$ are computed from the samples. One bank $i \in \mathcal{N}$ is uniformly sampled at random; its external asset holdings $r_{i}$ and $s_{i}$ are set to zero. This corresponds to a local shock to a single bank. For the resulting scenario, the greatest price-payment equilibrium and the corresponding number of defaulting firms is calculated. The simulation is repeated a large number of times, and sample averages and standard deviations are computed. The exact number of the simulations that were conducted is mentioned below for each case study. We use the following notation: Parameters corresponding to a basic Eisenberg-Noe model are denoted by EN, B signifies the incorporation of bankruptcy costs, $C$ cross-holdings, and $F$ fire sales.

\section{Separate effects}

First, we focus on the separate impact of individual model ingredients, leaving all other parameters as in the EN model. The section "Joint effects" investigates joint effects.

Bankruptcy costs and fire sales As expected, both bankruptcy costs and fire sales amplify the threat of contagion to the system. This is shown in Fig. 1.

Increasing bankruptcy costs, i.e., decreasing $\alpha$ and $\beta$, increases the number of defaults quite quickly, as shown by Fig. 1a. Part (b) of Fig. 1 shows a similar phenomenon when both fire sales parameters are increased. Additionally, a clear threshold effect can be observed that separates an area of many defaults from an area of few defaults. For low parameter values of $\rho$ and $\gamma$, the financial system exhibits the EN number of defaults (around 11). Increasing $\rho$ and $\gamma$ beyond a

\footnotetext{
${ }^{4}$ The choice of $c_{\Pi}=0.15$ is consistent with empirical findings for developed countries reported in Upper (2007) (with data from 2005), in Drehmann and Tarashev (2011) for the 20 largest internationally active banks, and with integration values for European countries computed in Glasserman and Young (2015).
} 

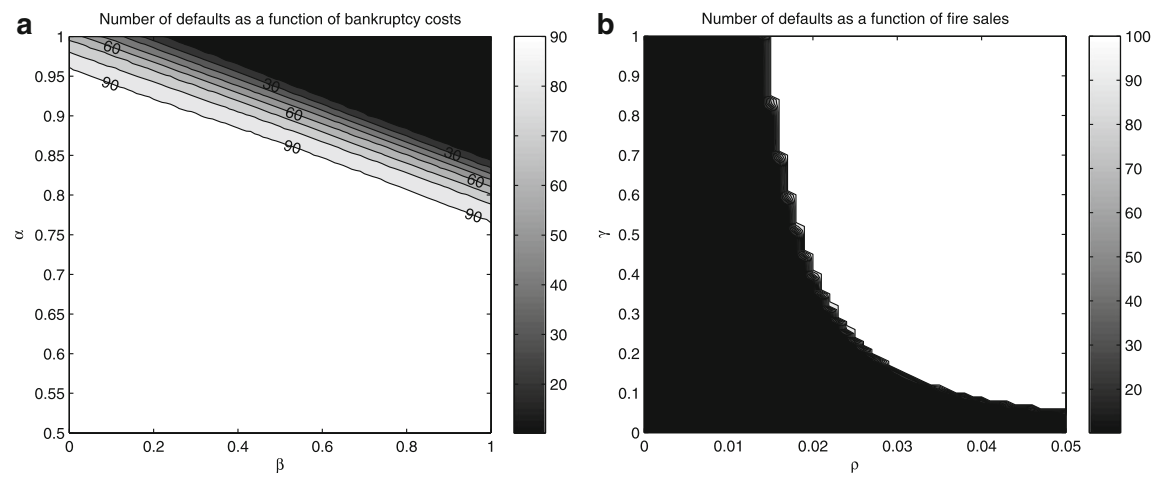

Fig. 1 Contour plots of the number of defaults for $n=100$ banks as a function of a bankruptcy costs and b fire sales, averaged over 1000 simulations of $\Pi$. The simulation procedure is explained in Remark 4.1

certain threshold boundary causes defaults of all banks in the system. The threshold curve can approximately be described by the following power-law function: $\rho=\exp (-4.3183) \cdot \gamma^{-0.4528}$.

From a policy point of view, these findings have two important implications:

(i) Bankruptcy costs increase the instability of the financial system significantly. These costs are mainly incurred due to the impaired operations of financial institutions in default. Administrative and legal expenses increase significantly for such institutions. Policies that improve the efficiency of managing defaults and restructuring institutions would mitigate the consequences of financial crises. This could, for example, be achieved by limiting the complexity of financial products and the operations of financial institutions. Another promising instrument are last wills of financial institutions, approved by the regulator during their lifetime, that simplify the processes in default.

(ii) Illiquidity, i.e., the joint consequences of limited funding and price impact, decreases market stability. When markets dry up, the value of financial institutions that need short-term funding might be significantly impaired. Quantitative easing would, in this case, be an appropriate instrument to stabilize the banking sector. This should include the purchase of temporarily illiquid assets.

The simulations also exhibit a sharp boundary between the regimes with few and many defaults. This indicates that regulatory policies should aim for substantial safety margins in order to create a resilient system.

Cross-Holdings Cross-holdings significantly impact the number of defaults. This has implications for the policies of regulators that we will discuss below.

Integration $c$ generally reduces the expected number of defaults. The dependence on $c$ is, however, non-monotonic. As shown in Fig. 2a, increasing $c$ to approximately 0.8 decreases their number at a nearly constant rate; beyond this point the number of defaults increases again. A second observation concerns diversification. For $d \geq 10$, the average number of defaults is largely independent of the value of $d$. This is also confirmed in Fig. 2 b. 

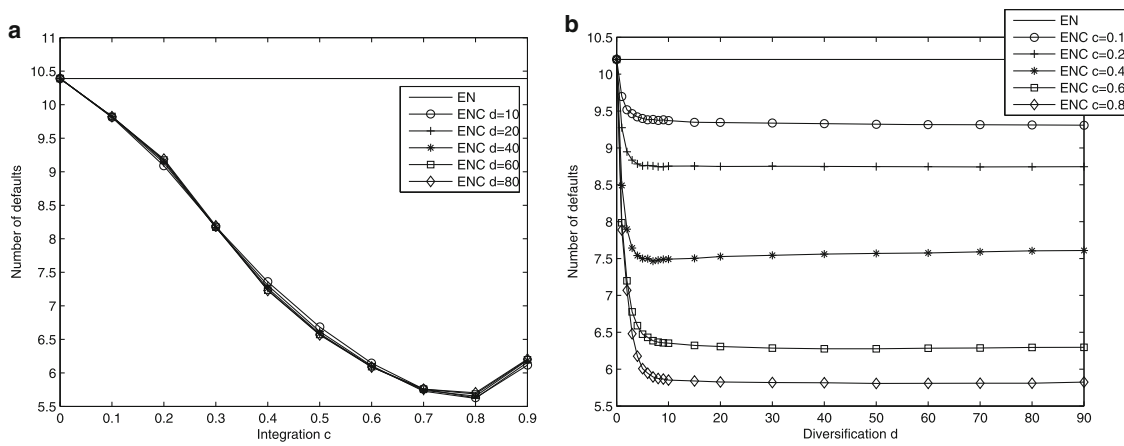

Fig. 2 Number of defaults for $n=100$ banks as a function of $\mathbf{a}$ integration and $\mathbf{b}$ diversification of the cross-holdings matrix $C$, realized fraction of cross-holdings' market value $\kappa=0.8$, averaged over 100 simulations of $\Pi$, each averaged over 100 simulations of $C$. The simulation procedure is explained in Remark 4.1

In addition to integration and diversification, the number of defaults also depends on the realized fraction $\kappa$ of the market value of cross-holdings. Figure 3 presents the expected monotonic effect.

Cross-ownership in the banking sector may stabilize the financial system. However, this finding relies on the existence of a market with substantial demand for cross-holdings. Our results show that regulators and central banks might use crossholdings in order to stabilize financial markets during financial crises. Regulatory policies that provide incentives to cross-ownership in the financial market as well as

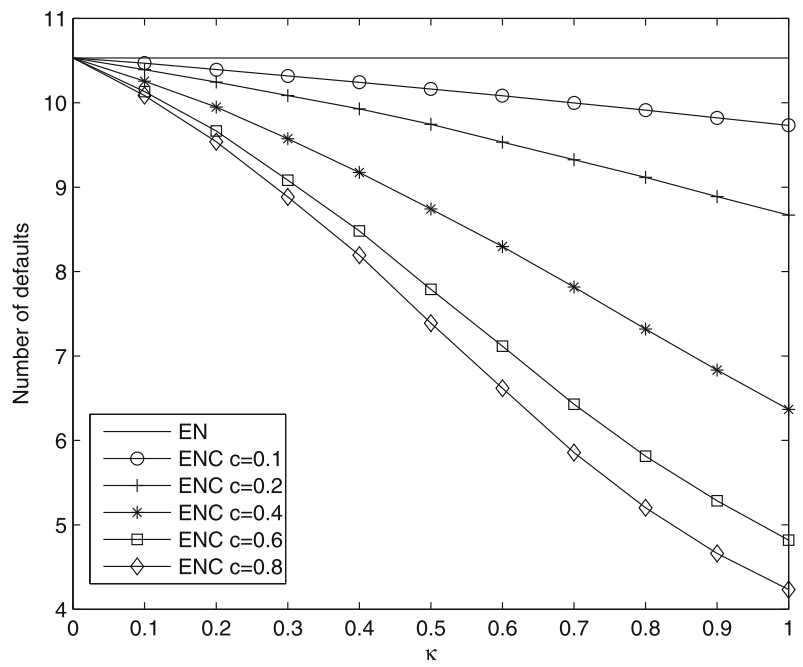

Fig. 3 Number of defaults as a function of the realized fraction $\kappa$ of cross-holdings' market value, varying integration and diversification $d=10$, averaged over 100 simulations of $\Pi$, each averaged over 100 simulations of $C$. The simulation procedure is explained in Remark 4.1 



Fig. 4 Standard deviation of the number of defaults for $n=100$ banks $\mathbf{a}$ as a function of both parameters of bankruptcy costs for 1000 simulations of $\Pi$, and $\mathbf{b}$ as a function of integration of the cross-holdings matrix $C$ for 100 simulations of $\Pi$, each averaged over 100 simulations of $C$. The simulation procedure is explained in Remark 4.1

a credible promise that these shares will be purchased, e.g., by the central bank would decrease systemic risk in our model.

The benefits of cross-holdings rely on the fact that they can be exchanged against cash. This effect becomes, of course, less significant if the realized fraction $\kappa$ is smaller. At the same time, there might be an inverse effect on the financial institution whose shares are sold. If a large sale of its shares decreases its equity price, its solvency is not directly affected: Solvency is a function of the book value of equitythe latter being computed from a market consistent balance sheet. The book value of equity may deviate from its market value. But a decreased equity price may increase the cost of capital and thereby negatively affect the bank's solvency indirectly. The closely related topic of the impact of equity valuation on credit supply to the real economy is, e.g., discussed in Boucinha et al. (2017).

Random fluctuations The preceding sections described the average behavior of the system. The actual outcomes, however, might significantly deviate from these averages. Two examples are provided in Fig. 4. The figures display the standard deviation ${ }^{5}$ of the number of defaults as (a) a function of the bankruptcy costs parameters $\alpha$ and $\beta$, and (b) as a function of the level $c$ of integration of the cross-holdings matrix.

A comparison of Figs. 4a and 1a leads to the following observation: For values of $\alpha$ and $\beta$ that lead to outcomes of either a very low number of defaults (i.e., EN-level) or the total breakdown of the system, the corresponding standard deviation is relatively low. However, in the transition area between these regimes, we observe a very high standard deviation. We observed a similar behavior when analyzing fire sales: while regimes with a very low or a very high average number of defaults exhibited low standard deviations, regimes with an intermediate average number of defaults

\footnotetext{
${ }^{5}$ In the ENC scenario, we computed the standard deviation on the basis of the $\Pi$ samples and continue to average over the $C$ simulations, since the conditional variance given $\Pi$ is relatively low.
} 
were associated with significant fluctuations around the averages and thus with significant risk. A refined analysis ${ }^{6}$ shows that the empirical distribution of defaults is mainly concentrated on the extreme scenarios of few or many defaults. Medium levels of bankruptcy costs or fire sales may, on average, seem acceptable, but are in fact associated with an unstable financial system. This shows that gradual improvements of the efficiency of the operations of distressed banks or light quantitative easing do not lead to resilience. Regulatory rules and interventions must be sufficiently forceful in order to achieve the desired effect of creating a stable financial system.

In the case of cross-holdings, an analysis of the average number of defaults is not sufficient. Comparing Fig. 4b with Fig. 2a shows that increasing integration has an overall beneficial effect on the average number of defaults, but increases the standard deviation within the considered parameter range. Features of the network thus have a quite complex impact on how financial stability and instability spread within the system. Moreover, Fig. 4b demonstrates that increasing diversification increases the standard deviation, while diversification does almost not affect the average number of defaults for $d>10$. A diversification of $d=10$ (i.e., $\sqrt{n}$ ) seems to be an optimal level. It would be interesting to investigate if such a statement holds more generally. Regulatory incentives for cross-ownership in the banking sector must therefore be very carefully designed. This requires substantial future research on the exact magnitude of the impact of cross-holdings in real financial networks.

\section{Joint effects}

In the current section, we investigate the joint impact of network effects, bankruptcy costs, fire sales, and cross-holdings in our integrated model and study the robustness of the results of the previous section. We focus on the following three questions: (i) Is quantitative easing in the joint model an appropriate policy for limiting systemic risk? How is this affected by other model drivers? (ii) Are the results modified by the change of the order of liquidation? (iii) Do cross-holdings have a beneficial effect on the stability of the financial system if bankruptcy costs and fire sales are included in the model? Which regulatory policies are appropriate?

(i) First, consider an integrated financial system with $\alpha=0.9, \beta=0.9, c=$ $0.5, d=10$, and $\kappa=0.8$. The resulting average number of defaults as a function of the fire sales parameters is displayed in Fig. ${ }^{7}$ A comparison of Figs. $1 \mathrm{~b}$ and $5 \mathrm{a}$ reveals that the general structure of the influence of price impact is preserved if bankruptcy costs and cross-holdings are added. However, the number of defaults increases and the sharp transition between the area of few defaults and the area of the breakdown of the system disappears. Similar qualitative results were all also confirmed in additional case studies based on both the ENBF and ENCF models. The results indicate that quantitative easing

\footnotetext{
${ }^{6} \mathrm{We}$ include an analysis of the empirical distribution in the joint model in Fig. 8. These findings were also confirmed in multiple other case studies for other parameter constellations.

${ }^{7}$ Kinks in the boundary are numerical artifacts. Note that the simulations still took several days to complete on the RRZN computing cluster.
} 

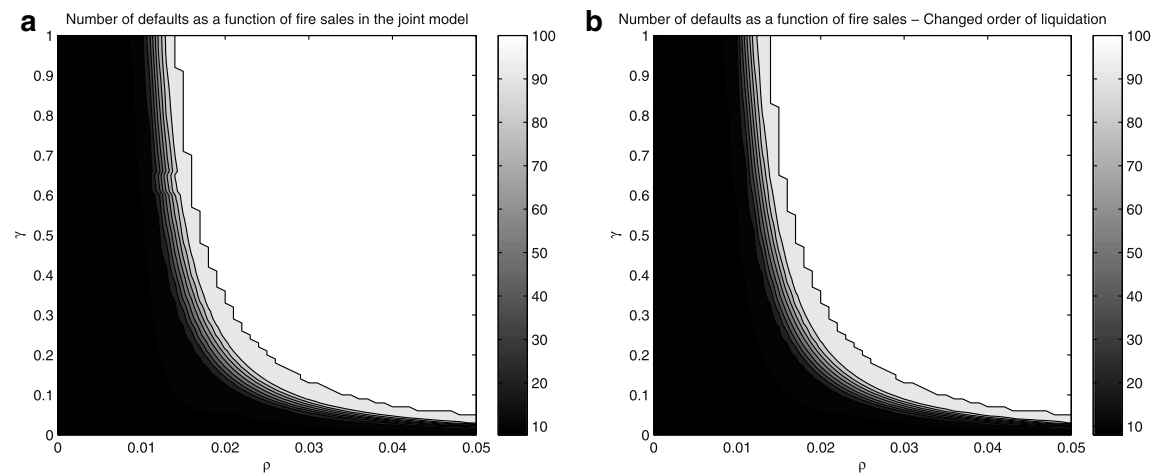

Fig. 5 Contour plot of the number of defaults for $n=100$ banks as a function of both parameters of fire sales, $\rho$ and $\gamma$, for $\alpha=0.9, \beta=0.9, c=0.5, d=10, \kappa=0.8, \mathbb{I}=\mathbf{1}$ in $\mathbf{a}$ and $\mathbb{I}=\mathbf{0}$ in $\mathbf{b}$, averaged over 100 simulations of $\Pi$, each averaged over 100 simulations of $C$. The simulation procedure is explained in Remark 4.1

continues to be a suitable instrument in order to contain the number of defaults in this case.

(ii) Second, Figs. $5 \mathrm{~b}$ and 6 deal with the impact of changing the order of liquidation on the number of defaults and the price of the illiquid asset as a function of the fire sales parameters $\rho$ and $\gamma$. These figures show that, regardless of whether cross-holdings or illiquid asset shares are liquidated first, the observed behavior is very similar.

(iii) Third, we analyze the effects of integration of cross-holdings in the joint model. This leads to rather complex, but very interesting features. We investigate a network model with $d=10, \kappa=0.8, \beta=0.9, \gamma=0.2, \rho=0.02$, and varying $\alpha$.
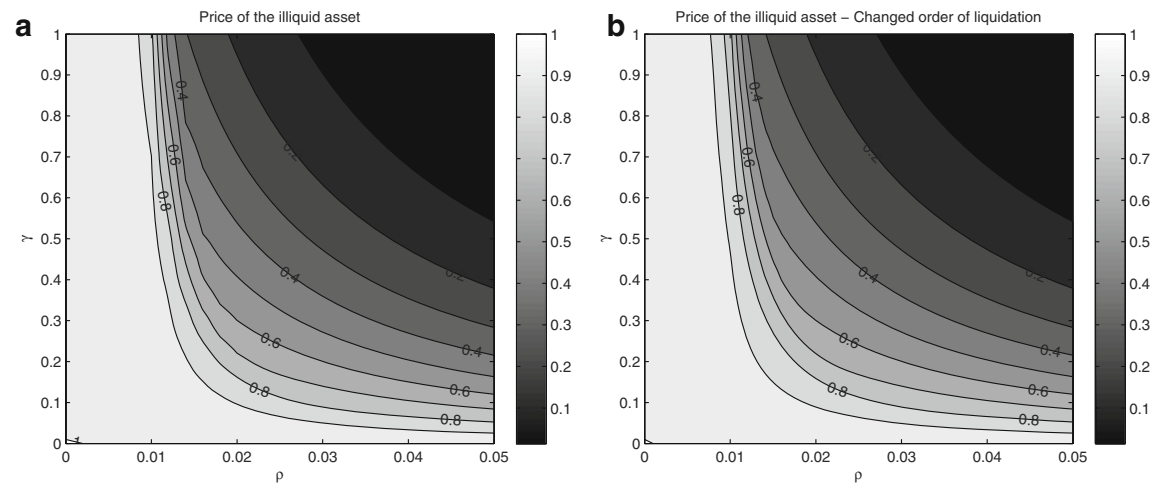

Fig. 6 Contour plot of the price of the illiquid asset for $n=100$ banks as a function of both parameters of fire sales, $\rho$ and $\gamma$, for $\alpha=0.9, \beta=0.9, c=0.5, d=10, \kappa=0.8, \mathbb{I}=\mathbf{1}$ in $\mathbf{a}$ and $\mathbb{I}=\mathbf{0}$ in $\mathbf{b}$, averaged over 100 simulations of $\Pi$, each averaged over 100 simulations of $C$. The simulation procedure is explained in Remark 4.1 
Within the considered parameter range, increasing integration decreases the average number of defaults, cf. Fig. 7a. The non-monotonicity observed in Fig. 2a disappears. As expected, the higher the realized fraction $\alpha$, the lower the number of defaults. However, for $\alpha \leq 0.8$ cross-holdings cannot prevent the total breakdown of the system. Observe that for the chosen value of $\beta=0.9$, the value $\alpha=0.8$ corresponds roughly to the critical boundary between the regimes of a very high $(\alpha \leq 0.8)$ and a very low $(\alpha \geq 0.9)$ number of defaults in Fig. 1a.

Figure $7 \mathrm{~b}$ displays standard deviations. These are comparatively small for extreme regimes of the default count statistics, i.e., $\alpha \in\{0.8,0.85,0.95\}$. For regimes with an intermediate average number of defaults, i.e., $\alpha \in$ $\{0.9,0.925\}$, the standard deviations are large. For fixed $\alpha \leq 0.9$, the standard deviation increases as a function of integration $c$; for $\alpha \geq 0.925$, the standard deviation is decreasing.

This different behavior can easily be understood when analyzing the empirical cumulative distribution functions (CDFs) of the number of defaults. It turns out that the distributions of the numbers of defaults are very close to Bernoulli distributions. In this case, the standard deviation is maximal for a success probability of 0.5 and monotonously decreasing if the success probability is either increased or decreased. As illustrated in Fig. 8, for $\alpha=0.9$, the distribution of defaults for $c=0.3$ roughly corresponds to a success probability of $0.2-0.3$; for $c=0.7$, to a success probability of $0.3-0.4$. Thus, the standard deviation increases in $c$. In contrast, for $\alpha=0.925$, the distribution of defaults for $c=0.3$ roughly corresponds to a success probability of $0.5-0.6$; for $c=0.7$, to a success probability of $0.7-0.8$. Thus, the standard deviation decreases in $c$.

The approximate Bernoulli distributions are supported by the EN number of defaults and a total breakdown of the system. This shows that the system essentially randomizes between extreme states. While the probability of the negative outcome can be controlled by cross-holdings for the chosen level of
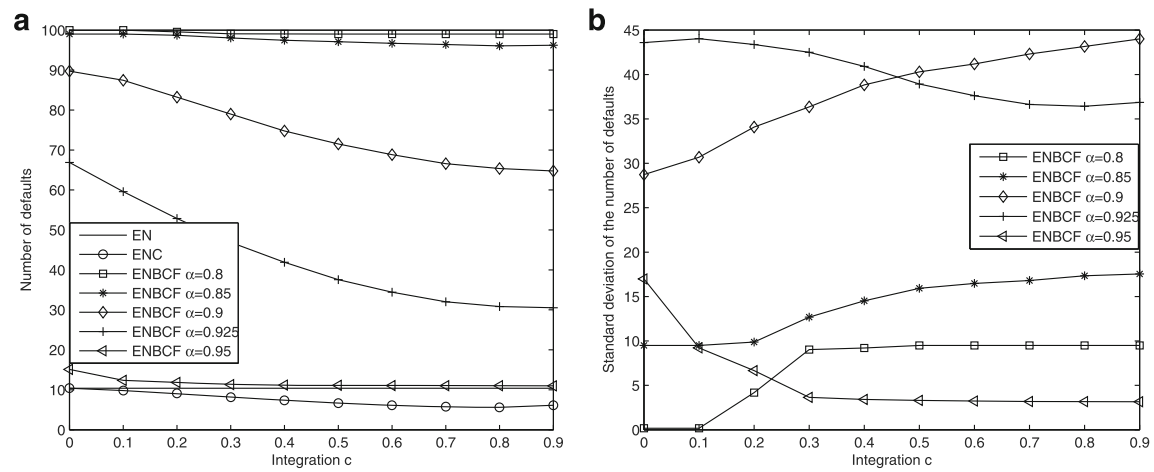

Fig. 7 Average a and standard deviation $\mathbf{b}$ of the number of defaults for $n=100$ banks as a function of integration $c$ for $d=10$, and for different bankruptcy costs parametrized by $\alpha$ in an integrated financial system with $\beta=0.9, \gamma=0.2, \rho=0.02, \kappa=0.8, \mathbb{I}=\mathbf{1}$ averaged over 100 simulations of $\Pi$, each averaged over 100 simulations of $C$. The simulation procedure is explained in Remark 4.1 

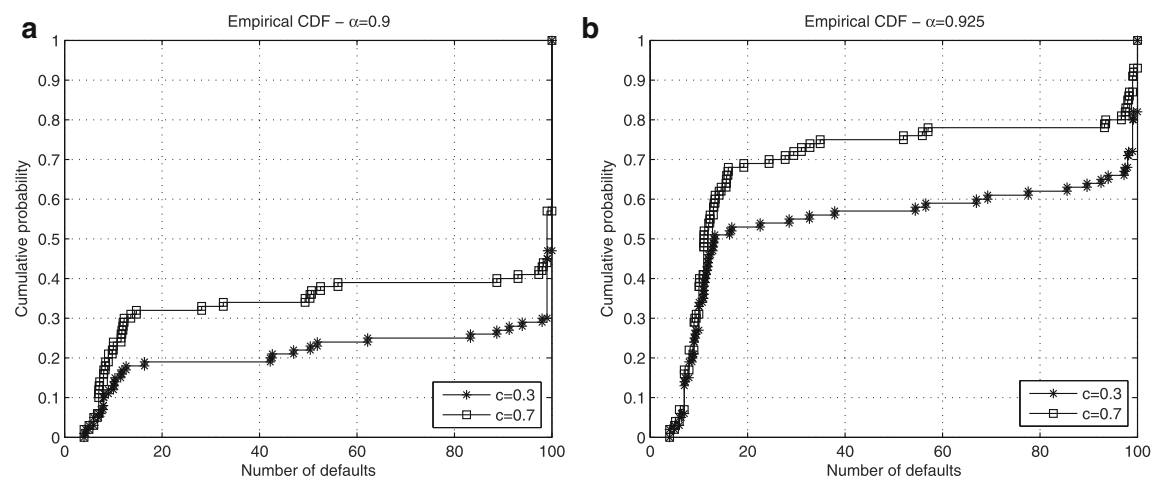

Fig. 8 Empirical cumulative distribution functions of the number of defaults in 100 simulations of the relative liabilities matrix $\Pi$ each averaged over 100 simulations of $C$, for two integration values $c=0.3$ and $c=0.7$ for $\alpha=0.9$ (a) and $\alpha=0.925$ (b)

bankruptcy costs and fire sales, the number of defaults in this adverse scenario is not mitigated.

From a policy point of view, our numerical case studies again indicate the fundamental role played by bankruptcy costs, emphasizing the importance of efficient procedures for managing defaults and restructuring institutions. Regulators should thus implement policies that lower the costs of bankruptcy. Moreover, if bankruptcy costs are not too large, a higher integration $c$ of crossholdings decreases both the average number of defaults and their variance. More integrated systems are thus more resilient. These results, of course, rely on the existence of a sufficiently deep and liquid market for cross-holdings. If this market dries up during a crisis, central banks might buy cross-holdings or provide guarantees for their purchase in order to stabilize the financial system. In our model, such a policy, however, does not seem to be efficient anymore if bankruptcy costs are too high.

\section{Capital adequacy ratios}

Capital requirements are a powerful instrument for the regulation of financial institutions. We investigate these in the section "Capital requirements" in more detail. For surveys of the literature on monetary risk measures, we refer to Föllmer and Schied (2011) and Föllmer and Weber (2015). A less sophisticated approach than monetary risk measures are capital adequacy ratios (CAR) based on risk-weighted assets. In the current section, we show in the context of our model that these are not always well-adopted for regulatory purposes.

We consider a stylized definition of CAR and ignore cross-holdings. For each bank $i$, capital $C_{i}$ is computed as the sum of external asset holdings $e_{i}$ and promised interbank holdings $\sum_{j \in \mathcal{N}} \Pi_{j i} \bar{p}_{j}$ less its liabilities $\bar{p}_{i}$. We assume that risk-weights ${ }^{8}$

\footnotetext{
${ }^{8}$ The specific risk-weight that is chosen is irrelevant to our findings.
} 
are $100 \%$ and calculate risk-weighted assets $R A_{i}$ of bank $i$ as the sum of its illiquid assets $s_{i}$ and its interbank holdings $\sum_{j \in \mathcal{N}} \Pi_{j i} \bar{p}_{j}$. The capital adequacy ratio of bank $i$ is defined by $C A R_{i}=C_{i} / R A_{i}$.

For a model including bankruptcy costs and fire sales, but no cross-holdings (ENBF), Fig. 9 displays the level sets of the lowest capital adequacy ratio in the banking system, $C A R$, as a function of the buffer $\delta$ and the proportion $\rho$ of the illiquid asset. At the same time, the boundary between the extreme default scenario (lower right corner) and few defaults (upper left corner) is shown.

A sufficiently high CAR can indeed prevent default cascades. However, in our case study, CAR appears to be an inefficient regulatory tool that does not properly measure the driving forces behind extreme default scenarios. Apparently, the barrier between many and few defaults and the level sets of CAR are not collinear. While for small values of $\rho$ a small CAR is sufficient, for large values of $\rho$ a large CAR is necessary in order to stabilize the system. The results indicate that regulators should base capital regulation on more sophisticated statistics than risk-weighted assets.

\section{Core-periphery random networks}

So far, we considered homogeneous random network topologies. Recent research on financial networks, however, suggests that core-periphery network models capture the structure of the interbank market (see, e.g., Craig and von Peter (2014) for the German interbank market, and van Lelyfeld and in't Veld (2014) for the Netherlands):

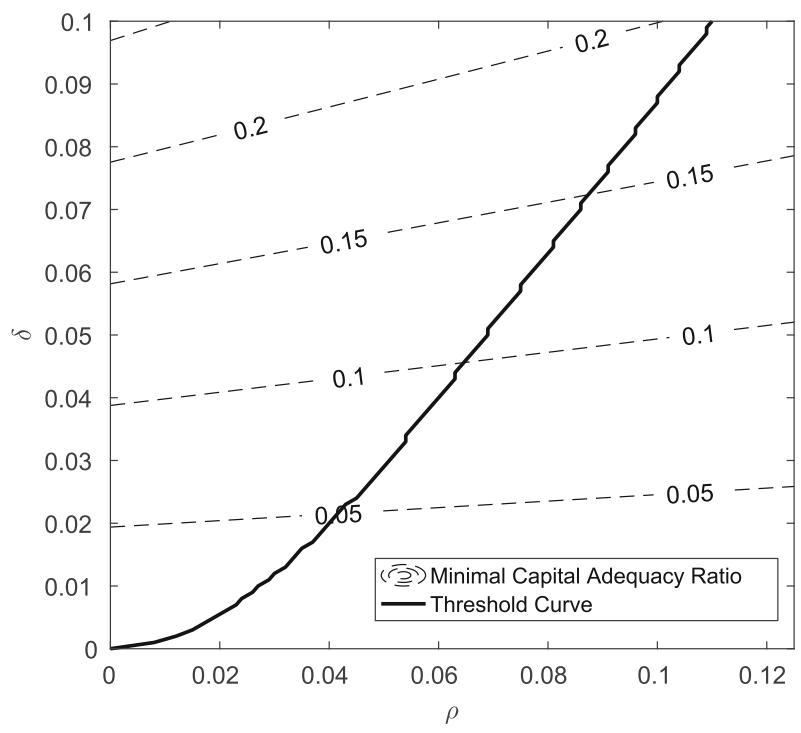

Fig. 9 Level sets of the lowest capital adequacy ratio in the banking system $C A R:=\min _{i \in \mathcal{N}} C A R_{i}$ as a function of the buffer $\delta$ and the proportion $\rho$ of the illiquid asset in an ENBF-model with $\alpha=0.925, \beta=0.9$, and $\gamma=0.2$. The solid line is the boundary between many (lower right) and few (upper left) defaults. Results are averaged over 100 simulations of $\Pi$. The simulation procedure is explained in Remark 4.1 
These consist of a few highly connected nodes (making up the core) and a larger number of only sparsely connected nodes (referred to as the periphery). In this section, we simulate the relative liabilities matrix $\Pi$ as a core-periphery random network and observe how this affects the impact of bankruptcy costs, cross-holdings, and fire sales on the number of defaults.

\section{Simulation methodology}

We divide the set of banks $\mathcal{N}$ into a subset of core banks, $\mathcal{C}$, and a subset of periphery banks, $\mathcal{P}$, with cardinalities $n^{C}$ and $n^{P}$, with $n^{C}+n^{P}=n$, respectively. We assume that a core-periphery relative liabilities matrix $\Pi$ can be represented by a random block matrix

$$
\Pi=\left(\begin{array}{ll}
C C & C P \\
P C & P
\end{array}\right) \in \mathbb{R}^{n \times n},
$$

where, for example, the block $C P \in \mathbb{R}^{n^{C} \times n^{P}}$ represents the core banks' liabilities towards the periphery banks. This matrix is constructed as follows.

1. Adjacency matrix: We first simulate an adjacency matrix $A \in \mathbb{R}^{n \times n}$, using exogenously specified connection probabilities $p^{C C}, p^{C P}, p^{P C}$, and $p^{P P}$ for each block.

2. Weights: Second, we fix the value of total liabilities $\ell$ of all banks. A fraction $c_{\Pi}$ is allocated to the total interbank liabilities; the remaining share models external liabilities that are uniformly distributed among all banks, i.e., $l_{i}=\frac{\left(1-c_{\Pi}\right) \cdot \ell}{n}$, $i \in \mathcal{N}$. Total interbank liabilities $c_{\Pi} \cdot \ell$ are allocated to the four matrix blocks in fractions of $x^{C C}, x^{P C}, x^{C P}$, and $x^{P P}$ with $x^{C C}+x^{P C}+x^{C P}+x^{P P}=1$. The resulting interbank liabilities per block are uniformly distributed among all existing links within the block. This is, denoting by $l^{C P}=\sum_{i \in \mathcal{C}} \sum_{j \in \mathcal{P}} A_{i j}$ the random number of total links in the $C P$-block, the corresponding entries of the nominal liabilities matrix $L$ are $L_{i j}=\frac{{ }^{C}{ }^{C P} \cdot c_{\Pi} \cdot \ell}{l^{C P}}, i \in \mathcal{C}, j \in \mathcal{P}$, if $A_{i j}>0$, and $L_{i j}=0$, otherwise. The other blocks are computed analogously. Finally, with $\bar{p}_{i}=\sum_{j \in \mathcal{N}} L_{i j}+l_{i}$, the entries of the relative liabilities matrix $\Pi$ are given by $\Pi_{i j}=\frac{L_{i j}}{\bar{p}_{i}}$.

As in the other case studies, we set $n=100, c_{\Pi}=0.15$, and $\ell=100$. In addition, following Craig and von Peter (2014) who analyzed data from the German interbank market, we choose core-periphery parameters

$$
\begin{aligned}
p^{C C} & =0.66, p^{C P}=0.15, p^{P C}=0.07, p^{P P}=0.001, \\
x^{C C} & =0.35, x^{C P}=0.16, x^{P C}=0.47, x^{P P}=0.02 .
\end{aligned}
$$

We fix the number of core banks as $n^{C}=10 .{ }^{9}$ The simulation methodology is analogous to the section "Simulation methodology". Note, however, that simulation results will depend on whether a core or periphery bank is hit by an initial shock.

\footnotetext{
${ }^{9}$ This number is consistent with the empirical results of van Lelyfeld and in't Veld (2014), who find a core of around 10 banks in the Dutch interbank network, where the total number of banks is around our chosen number of 100 .
} 



Fig. 10 Contour plots of the number of defaults for $n=100$ banks as a function of a bankruptcy costs and $\mathbf{b}$ fire sales, conditional on an initial core shock, averaged over 100 simulations of $\Pi$, simulated as a core-periphery random network. The simulation procedure is explained in Remark 4.1

\section{Results}

We focus on three case studies: the separate impact of a) bankruptcy costs and b) fire sales as well as c) the joint impact of all ingredients while varying the parameters that govern the fire sales.

Figure 10 shows the effects of bankruptcy costs and fire sales in the core-periphery network, given that initially a core bank is shocked. ${ }^{10}$ The qualitative results for an initial periphery shock are similar; however, the total average number of defaults tends to be higher as an initially shocked core bank may survive the shock due to a high and possibly stabilizing level of interconnectedness.

Comparing Figs. 10 to 1, we observe that bankruptcy costs and fire sales have a similar impact as in the Erdös-Rényi network: as expected, an increase in the number of defaults for increasing bankruptcy costs and fire sales; a clear threshold boundary separating an area of many defaults from an area of few defaults. The exact numbers are, of course, different. For example, in Fig. 10 (a) the impact of parameter $\beta$ on defaults is stronger than before.

Figure 11 shows the average number of defaults as a function of both fire sales parameters in an ENBCF core-periphery model given that initially a core bank's external assets lose their total value.

Again, our observations resemble the findings within Erdös-Rényi random networks.

The considered core-periphery structure was calibrated to German interbank market data and presents a good description of a real world interbank market. Overall, the above comparisons indicate that the results we obtained are qualitatively similar to those within simpler Erdös-Rényi random networks. This shows that the simplified setting is already representative and relevant for the analysis of systemic risk.

\footnotetext{
${ }^{10}$ Observe that rough boundaries are artifacts of the numerical implementation. Still, computing time on the RRZN cluster was several days.
} 


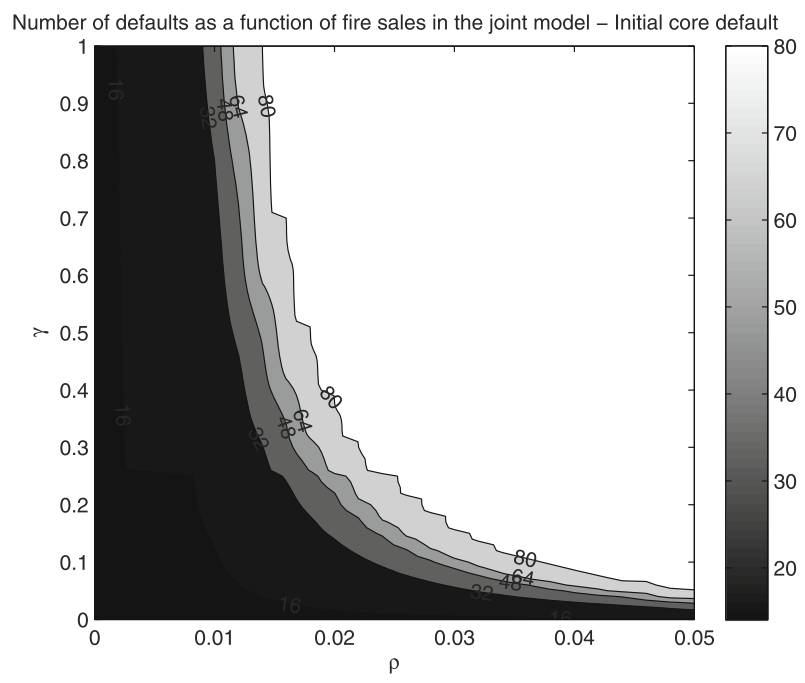

Fig. 11 Contour plot of the number of defaults for $n=100$ banks as a function of fire sales in the joint model $(\alpha=0.9, \beta=0.9, c=0.5, d=10, \kappa=0.8, \mathbb{I}=\mathbf{1})$, conditional on an initial core default, averaged over 100 simulations of $\Pi$, simulated as a core-periphery random network, each averaged over 100 simulations of $C$ simulated as an Erdös-Rényi random network. The simulation procedure is explained in Remark 4.1

The policy implications discussed in the context of Erdös-Rényi random networks remain valid for the considered core-periphery structure.

\section{Multi-layer networks}

Our model can be extended to more than two layers and is capable of modeling heterogenous agents. We will illustrate this with three types of agents: banks, depositors, and borrowers. Depositors hold deposits at banks. Borrowers receive credit from the banks that act as intermediaries. For simplicity, we neglect bankruptcy costs and cross-holdings and assume that the banking system consists of 20 banks that form an Erdös-Rényi random network with $d_{\Pi}=2$ and $\delta=0.2$. We add depositors and borrowers to the system. The total liabilities of all banks (to other banks and to the depositors) within the considered time-period are normalized to the total number of banks, i.e., 20. Liabilities within the banking system are set to $15 \%$ of total liabilities, i.e., $c_{\Pi}=0.15$.

The remaining $85 \%$ are liabilities to depositors. The number of depositors is 90 , and each depositor is linked to exactly two banks that are uniformly sampled at random. Each link is associated with the same liability. We assume that all liabilities are immediately due. This scenario can be interpreted as a bank run.

There are 90 borrowers outside the banking system. Each borrower is linked to two banks that are sampled uniformly at random. Borrowers' short-term liabilities over the considered time-period are 1/100 per link. Borrowers hold external assets 
which amount to $1+\delta$ of their total short-term liabilities; $40 \%$ of these are allocated to the illiquid asset.

All quantities are then computed as in the section "Simulation methodology". Figure 12 displays the average defaults in the banking system as a function of the fire sales parameters. Qualitatively, the graph resembles previous results and emphasizes their robustness. This shows again that quantitative easing can stabilize the banking system.

\section{Capital requirements}

Another important regulatory tool are capital requirements for banks. In financial networks, the financial situation of a bank, of course, does not only depend on its own capital endowment, but also on the capital of other financial institutions with which it interacts directly or indirectly. Within a core-periphery network, we discuss the role of capital on the basis of a framework suggested in Feinstein et al. (2017). We refer to this paper for further details on systemic risk measures.

In the current case study, all simulations are conducted according to the same methodology as described in the section "Simulation methodology". However, we introduce two further parameters $k^{C}$ and $k^{P}$ which signify additional capital on top of the originally computed external assets $e$ that is held by the core and periphery banks, respectively. As before, the updated amount of the external assets is then divided into liquid and illiquid assets according to the parameter $\rho$, and the simulations are run.

In order to evaluate the effect of the additional capital encoded by the vector $k=$ $\left(k^{C}, k^{P}\right)$ on the system, we use the following approach. Given $k$, we compute the

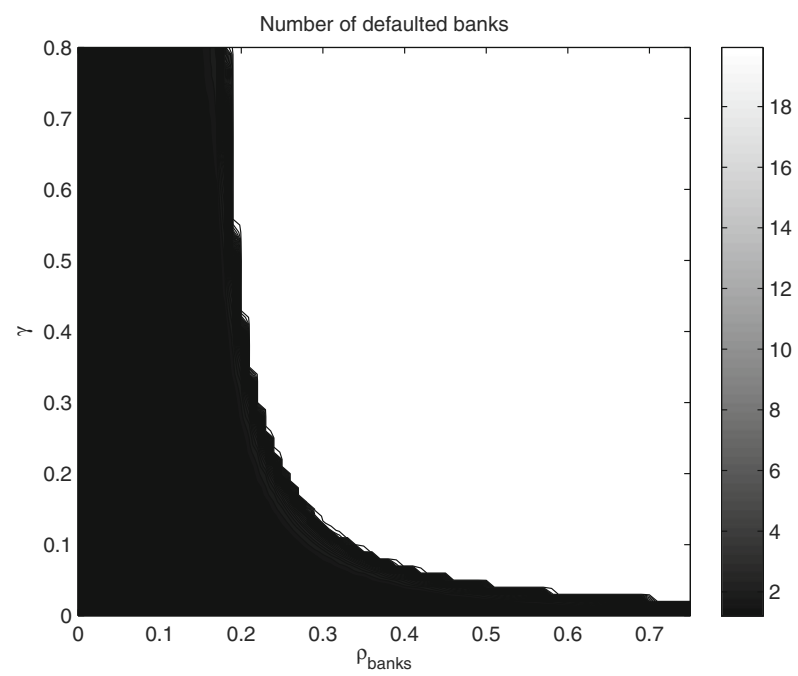

Fig. 12 Contour plot of the average number of bank defaults as a function of fire sales parameters $\rho_{\text {banks }}$ and $\gamma$ in an ENF-model, averaged over 100 simulations of $\Pi$ and periphery link choices. The simulation procedure is explained in Remark 4.1 
greatest clearing vector $p^{*}(k)$ in our system. The clearing vector is a random quantity. The total random losses from direct liabilities are then given by:

$$
L(k)=\sum_{i \in \mathcal{N}}\left(1-\sum_{j \in \mathcal{N}} \Pi_{i j}\right) \cdot\left(p_{i}^{*}(k)-\bar{p}_{i}\right) .
$$

Observe that losses are considered negative. If there are no losses, $L(k)$ is equal to 0 . Since financial institutions provide services to society, we assume that a regulator accepts a small loss or cost of up to $t$, but higher losses only with probability $\alpha$. We define the set $R$ of all $k=\left(k^{C}, k^{P}\right) \in \mathbb{R}^{2}$ that are compatible with this requirement:

$$
R:=\left\{k \in \mathbb{R}^{2}: P(L(k) \leq-t) \leq \alpha\right\}=\left\{k \in \mathbb{R}^{2}: V @ R_{\alpha}(L(k)) \leq t\right\} .
$$

The set $R$ is a systemic risk measure in the sense of Feinstein et al. (2017).

We now consider an ENBF model and a core-periphery network as described in the section "Simulation methodology" and choose $t=1 / 20$ and $\alpha=10 \%$. Figure 13a displays the boundary of the set-valued systemic risk measures for varying fractions $\rho$ of the illiquid asset without bankruptcy costs. Note that by definition, the systemic risk measure is an upper set in the sense that all points above the boundary are acceptable. As expected, we find that the higher the proportion of the illiquid asset in the external asset portfolio, i.e., the higher the $\rho$, the higher the capital requirement for both core and periphery banks is. The figure also shows that capital requirements tighten in a similar way for both core and periphery banks if the proportion of the illiquid asset is increased. This indicates, that according to our model, regulators should pay attention to the capital requirements of all banks if price impact is an important factor during crises.

Figure (b) includes bankruptcy costs for varying $\beta$ with $\rho=0.5$ fixed. While the capital requirements for the periphery banks are barely affected by decreasing $\beta$, the requirements for the core banks increase strongly. Since core banks are more connected than periphery banks, the impact of defaults and resulting
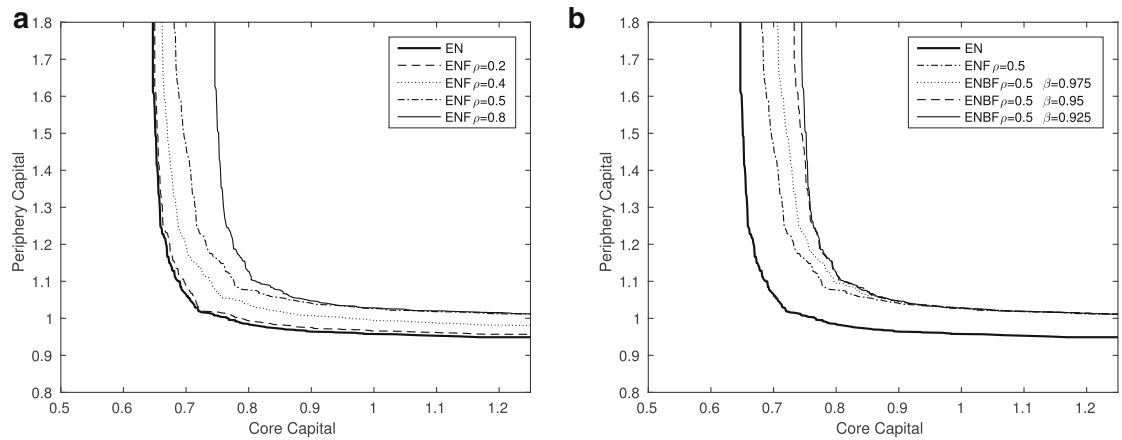

Fig. 13 Capital requirements for core and periphery banks as a function of fire sales (varying $\rho$ and fixed $\gamma=0.2$ ) a separately and $\mathbf{b}$ together with different levels of bankruptcy costs (varying $\beta$ and fixed $\alpha=1$ ) 
interbank bankruptcy costs on core banks is more significant than on periphery banks. In the context of the chosen model, a regulator would be well advised if she particularly focused on strengthening the capital endowments of core banks. These can serve as a buffer that reduces systemic risk even if considerable bankruptcy costs are present.

\section{Conclusion}

The paper presents a comprehensive model of a financial system that integrates network effects, bankruptcy costs, fire sales, and cross-holdings. For the integrated financial market, we prove the existence of a price-payment equilibrium and design an algorithm for the computation of the greatest and the least equilibrium. The number of defaults corresponding to the greatest price-payment equilibrium was analyzed in several comparative case studies for both simple Erdös-Rényi and more realistic core-periphery and multi-layer random networks:

(i) Systemic risk was studied by shocking the system and computing the average number of defaults, its variance, and distribution. Outcomes are centered on extreme scenarios. The risk of extreme adverse events is present, even if averages indicate a relatively safe system. Regulatory policies should provide substantial safety margins in order to guarantee stability.

(ii) Fire sales strongly increase systemic risk, while cross-holdings may improve the resilience of the banking sector. Central banks might mitigate the risk of default cascades by purchasing illiquid assets and cross-holdings. Quantitative easing strengthens the system.

(iii) Bankruptcy costs are a main driver of systemic risk. Regulators should improve the efficiency of bankruptcy procedures and limit the associated deadweight losses. Policies might include reducing the complexity of financial products as well as operational procedures and requiring last wills of financial institutions.

(iv) Capital requirements are a powerful instrument, but capital adequacy ratios based on risk-weighted assets are an extremely rough measure of systemic risk. Instead, modern systemic risk measures that use capital efficiently could be implemented.

(v) We analyzed different interbank network structures and heterogeneous business models. Our qualitative results were robust. Quantitative predictions, however, require a precise specification of all driving mechanisms.

The suggested model can be used as a framework for testing the impact of regulatory policies and their robustness. It can also provide insights into the significance of the financial market architecture for systemic risk, e.g., the pros and cons of CCPs. From a statistical point of view, our comparative statics show that default cascades can be triggered by a combination of various mechanisms. In particular, bankruptcy costs and fire sales exhibit similar consequences. Their statistical estimation is a challenging issue for future research that requires further data on historical bankruptcy procedures and price impact during crises. 


\section{Proofs}

\section{Proof of Lemma 2.2.}

(a) This follows directly from Banach's fixed-point theorem applied to the function $\Psi$.

(b) We prove that the essential net worths vector is increasing in the payments $p$. The corresponding claim for the illiquid asset's price $q$ can be proven analogously. To simplify the notation, we suppress the dependence on $q$ and write $w^{*}(p)$ instead of $w^{*}(p, q)$ in the following. Now, for each given payment vector $p$, we define the following recursive sequence: Starting with $w^{(0)}(p):=$ $r+s q+\Pi^{T} p-\bar{p}$, we set $w^{(n)}(p)=\Psi\left(w^{(n-1)}(p)\right)$, for $n=1,2, \ldots$ Due to part (a), this sequence converges with $\lim _{n \rightarrow \infty} w^{(n)}(p)=w^{*}(p)$.

For two given payment vectors $p^{1} \geq p^{2}$, it holds that $w^{(n)}\left(p^{1}\right) \geq w^{(n)}\left(p^{2}\right)$ for all $n$. We prove this statement by induction. For $n=0$,

$$
w^{(0)}\left(p^{1}\right)=r+s q+\Pi^{T} p^{1}-\bar{p} \geq r+s q+\Pi^{T} p^{2}-\bar{p}=w^{(0)}\left(p^{2}\right),
$$

since $p^{1} \geq p^{2}$. For the induction step, $n \rightarrow n+1$, it holds that

$$
\begin{aligned}
w^{(n+1)}\left(p^{1}\right) & =r+s q+\Pi^{T} p^{1}+\operatorname{diag}\left(\mu\left(p^{1}, w^{(n)}\left(p^{1}\right)\right) C^{T}\left(w^{(n)}\left(p^{1}\right) \vee \mathbf{0}\right)-\bar{p}\right. \\
& \geq r+s q+\Pi^{T} p^{2}+\operatorname{diag}\left(\mu\left(p^{2}, w^{(n)}\left(p^{2}\right)\right) C^{T}\left(w^{(n)}\left(p^{2}\right) \vee \mathbf{0}\right)-\bar{p}\right. \\
& =w^{(n+1)}\left(p^{2}\right),
\end{aligned}
$$

exploiting the induction hypothesis and the fact that $\mu_{i}(p, w)$ is by definition increasing in both components. Hence, $w^{(n)}\left(p^{1}\right) \geq w^{(n)}\left(p^{2}\right)$ for all $n$, and this yields

$$
w^{*}\left(p^{1}\right)=\lim _{n \rightarrow \infty} w^{(n)}\left(p^{1}\right) \geq \lim _{n \rightarrow \infty} w^{(n)}\left(p^{2}\right)=w^{*}\left(p^{2}\right) .
$$

\section{Proof of Lemma 3.1.}

(a) It holds by definition that $\Phi_{i}(p, q)=\chi_{i}(p, q) \geq 0$ and $\Phi_{i}(p, q) \leq \bar{p}_{i}$ for all $i=1, \ldots, n$, since the banks will pay at most their total liabilities. For $i=n+1$, the boundedness follows from the definition of the inverse demand function.

(b) Let $\left(p^{1}, q^{1}\right) \leq\left(p^{2}, q^{2}\right)$. We have that $\theta\left(p^{1}, q^{1}\right) \geq \theta\left(p^{2}, q^{2}\right)$, because the essential net worths vector is monotone in the price-payment pairs due to Lemma 2.2 (b). Hence,

$$
\Phi_{n+1}\left(p^{1}, q^{1}\right)=f\left(\theta\left(p^{1}, q^{1}\right)\right) \leq f\left(\theta\left(p^{2}, q^{2}\right)\right)=\Phi_{n+1}\left(p^{2}, q^{2}\right),
$$

since $f$ is monotonically decreasing. For $i=1, \ldots, n$, monotonicity follows from a case-by-case analysis extending the arguments of Rogers and Veraart (2013) and Amini et al. (2013). First, assume that bank $i$ is in default under $\left(p^{2}, q^{2}\right)$. This implies that it is in default under $\left(p^{1}, q^{1}\right)$ and that

$\Phi_{i}\left(p^{2}, q^{2}\right)=\alpha\left[r_{i}+s_{i} q^{2}\right]+\beta \eta_{i}\left(p^{2}, q^{2}\right) \geq \alpha\left[r_{i}+s_{i} q^{1}\right]+\beta \eta_{i}\left(p^{1}, q^{1}\right)=\Phi_{i}\left(p^{1}, q^{1}\right)$, 
due to Lemma 2.2 (b). Next, assume that bank $i$ does not default under $\left(p^{2}, q^{2}\right)$. Then, bank $i$ can either survive or default under $\left(p^{1}, q^{1}\right)$. In the first case, monotonicity of $\Phi_{i}$ directly follows from $\Phi_{i}\left(p^{2}, q^{2}\right)=\bar{p}_{i}=\Phi_{i}\left(p^{1}, q^{1}\right)$. In the second case, if bank $i$ defaults under $\left(p^{1}, q^{1}\right)$ but not under $\left(p^{2}, q^{2}\right)$, it follows that

$$
\Phi_{i}\left(p^{2}, q^{2}\right)=\bar{p}_{i}>r_{i}+s_{i} q^{1}+\eta_{i}\left(p^{1}, q^{1}\right) \geq \alpha\left[r_{i}+s_{i} q^{1}\right]+\beta \eta_{i}\left(p^{1}, q^{1}\right)=\Phi_{i}\left(p^{1}, q^{1}\right) .
$$

Here, the first inequality holds true, since bank $i$ defaults under $\left(p^{1}, q^{1}\right)$. The second inequality follows from $\alpha, \beta \leq 1$.

\section{Proof of Theorem 3.2}

Let $\mathcal{F}:=\left\{(p, q) \in[\mathbf{0}, \bar{p}] \times\left[q_{\min }, q_{0}\right] \mid \Phi(p, q)=(p, q)\right\}$ denote the set of fixed points of $\Phi$. Lemma 3.1 establishes that $\Phi$ is an increasing function on the complete lattice $\left\langle[\mathbf{0}, \bar{p}] \times\left[q_{\min }, q_{0}\right], \leq\right\rangle$ with the componentwise $\leq$-relation. Hence, Tarski's fixed-point theorem Tarski (1955, Theorem 1) states that $\mathcal{F}$ is not empty and, moreover, that $\langle\mathcal{F}, \leq\rangle$ constitutes a complete lattice itself. In particular, this yields the existence of a unique greatest and least element of $\mathcal{F}$. Since the elements of $\mathcal{F}$ constitute by definition the price-payment equilibria, this completes the proof.

\section{Proof of Theorem 3.5}

This proof is an extension of the proof provided by Rogers and Veraart (2013) and proceeds in three steps. First, we show that the sequence of price-payment pairs $\left(p^{(k)}, q^{(k)}\right)$ decreases. Second, we demonstrate that each pair in this sequence is larger than or equal to the greatest price-payment equilibrium. Third, we observe that when the algorithm terminates, the calculated price-payment pair equals the greatest price-payment equilibrium.

(i) For the first step, we claim that

$$
\left(p^{(k+1)}, q^{(k+1)}\right) \leq\left(p^{(k)}, q^{(k)}\right) \quad \forall k=0,1, \ldots, n-1 .
$$

We prove this statement by induction.

(B.S.) For the base step, $k=0$, we need to prove that $p^{(1)} \leq p^{(0)}=\bar{p}$ and that $q^{(1)} \leq q^{(0)}=q_{0}$. Regarding the payments, this clearly holds for all $i \in \mathcal{S}_{0}$, since then $p_{i}^{(1)}=\bar{p}_{i}=p_{i}^{(0)}$. Thus, it remains to examine the payments of the defaulting banks in $\mathcal{D}_{0}$ and the corresponding price of the illiquid asset, which are jointly given by the maximal solution to the Eqs. (4) and (5). In order to calculate this maximal solution, we propose the following recursive procedure: Start with $\left(x^{(0)}, y^{(0)}\right)$, where $x_{i}^{(0)}=p_{i}^{(0)}=\bar{p}_{i}$ for $i \in$ $\mathcal{D}_{0}$ and $y^{(0)}=q^{(0)}=q_{0}$, and define the sequence $\left(x^{(v)}, y^{(v)}\right)$ recursively by

$$
x_{i}^{(\nu+1)}=\alpha\left[r_{i}+s_{i} y^{(\nu)}\right]+\beta\left[\sum_{j \in \mathcal{D}_{0}} \Pi_{j i} x_{j}^{(\nu)}+\sum_{j \in \mathcal{S}_{0}}\left[\Pi_{j i} \bar{p}_{j}+\lambda C_{j i} \max \left(w_{j}^{*}\left(x^{(\nu)}, y^{(\nu)}\right), 0\right)\right]\right]
$$


for $i \in \mathcal{D}_{0}$ and

$$
y^{(v+1)}=f\left(\sum_{i \in \mathcal{D}_{0}} s_{i}+\sum_{i \in \mathcal{S}_{0}} \min \left(\frac{\zeta_{i}^{(0)}\left(x^{(v)}, y^{(v)}\right)}{y^{(v)}}, s_{i}\right)\right),
$$

with $\zeta_{i}^{(0)}(x, y)$ for all $i \in \mathcal{S}_{0}$ defined as in Eq. (6), substituting $k$ with 0 . Next, we have to show that

$$
\left(x^{(v+1)}, y^{(v+1)}\right) \leq\left(x^{(v)}, y^{(v)}\right) \quad \forall v=0,1, \ldots,
$$

which we will prove by induction. First, for the base case, $v=0$, we observe that for $i \in \mathcal{D}_{0}$ :

$$
\begin{aligned}
x_{i}^{(1)} & =\alpha\left[r_{i}+s_{i} y^{(0)}\right]+\beta\left[\sum_{j \in \mathcal{D}_{0}} \Pi_{j i} x_{j}^{(0)}+\sum_{j \in \mathcal{S}_{0}}\left[\Pi_{j i} \bar{p}_{j}+\lambda C_{j i} \max \left(w_{j}^{*}\left(x^{(0)}, y^{(0)}\right), 0\right)\right]\right] \\
& \leq r_{i}+s_{i} q^{(0)}+\sum_{j \in \mathcal{D}_{0}} \Pi_{j i} p_{j}^{(0)}+\sum_{j \in \mathcal{S}_{0}} \Pi_{j i} \bar{p}_{j}+\lambda \sum_{j \in \mathcal{S}_{0}} C_{j i} \max \left(w_{j}^{*}\left(p^{(0)}, q^{(0)}\right), 0\right) \\
& =r_{i}+s_{i} q^{(0)}+\sum_{j=1}^{n} \Pi_{j i} p_{j}^{(0)}+\lambda \sum_{j=1}^{n} C_{j i} \max \left(w_{j}^{*}\left(p^{(0)}, q^{(0)}\right), 0\right)<\bar{p}_{i}=p_{i}^{(0)}=x_{i}^{(0)} .
\end{aligned}
$$

Here, the first inequality is satisfied because $0 \leq \alpha, \beta \leq 1$. The second step follows from $p_{j}^{(0)}=\bar{p}_{j}$ on $\mathcal{S}_{0}$ and $w_{j}^{*}\left(p^{(0)}, q^{(0)}\right)<0$ for $j \in \mathcal{D}_{0}$. The last step holds according to the definition of $\mathcal{D}_{0}$ and due to the fact that $0 \leq \lambda \leq \mu_{i}\left(p^{(0)}\right) \leq 1$.

Moreover,

$$
\sum_{i \in \mathcal{D}_{0}} s_{i}+\sum_{i \in \mathcal{S}_{0}} \min \left(\frac{\zeta_{i}^{(0)}\left(x^{(0)}, y^{(0)}\right)}{y^{(0)}}, s_{i}\right) \geq 0
$$

by definition of $\zeta_{i}^{(0)}\left(x^{(0)}, y^{(0)}\right)$. Thus, since $f$ is monotonically decreasing and $f(0)=q_{0}$, it follows that $y^{(1)} \leq q_{0}=y^{(0)}$. Now, suppose that inequality (9) is satisfied up to some $v$. Then, one obtains:

$$
\begin{aligned}
& \zeta_{i}^{(0)}\left(x^{(v)}, y^{(v)}\right) \\
& =\max \left(\bar{p}_{i}-r_{i}-\sum_{j \in \mathcal{D}_{0}} \Pi_{j i} x_{j}^{(v)}-\sum_{j \in \mathcal{S}_{0}}\left[\Pi_{j i} \bar{p}_{j}+\mathbb{I}_{i} \lambda C_{j i} \max \left(w_{j}^{*}\left(x^{(v)}, y^{(v)}\right), 0\right)\right], 0\right) \\
& \geq \zeta_{i}^{(0)}\left(x^{(\nu-1)}, y^{(\nu-1)}\right),
\end{aligned}
$$

by the induction hypothesis and Lemma 2.2 (b). This yields $y^{(v+1)} \leq y^{(v)}$, again exploiting the induction hypothesis together with the fact that $f$ is monotonically decreasing.

Analogously, it follows from the recursive definition that $x_{i}^{(v+1)} \leq x_{i}^{(v)}$ for all $i \in \mathcal{D}_{0}$. Thus, the sequence continues to decrease for all $v$. Hence, 
the limit $(x, y):=\lim _{v \rightarrow \infty}\left(x^{(v)}, y^{(v)}\right)$ exists and solves Eqs. (4) and (5). Moreover, $(x, y)$ is the maximal solution to these equations by construction. This completes the base step of the induction argument for the proof of (8).

(I.S.) For the induction step, $k \rightarrow k+1$, we first observe that by the induction hypothesis we have that $\mathcal{D}_{k} \supseteq \mathcal{D}_{k-1}$ or, equivalently, $\mathcal{S}_{k} \subseteq \mathcal{S}_{k-1}$. Hence, for all $i \in \mathcal{S}_{k}: p_{i}^{(k+1)}=\bar{p}_{i}=p_{i}^{(k)}$. Thus, we have to investigate the payments of the defaulting banks and the corresponding price of the illiquid asset, defined by the maximal solution to Eqs. (4) and (5). Analogous to the base step, we propose the following recursive principle to calculate this maximal solution. Start with $\left(x^{(0)}, y^{(0)}\right)$, where $x_{i}^{(0)}=p_{i}^{(k)}$ for $i \in \mathcal{D}_{k}$ and $y^{(0)}=q^{(k)}$, and define $\left(x^{(v)}, y^{(v)}\right)$ by the obvious modification of the above recursive principle:

$x_{i}^{(\nu+1)}=\alpha\left[r_{i}+s_{i} y^{(\nu)}\right]+\beta\left[\sum_{j \in \mathcal{D}_{k}} \Pi_{j i} x_{j}^{(\nu)}+\sum_{j \in \mathcal{S}_{k}}\left[\Pi_{j i} \bar{p}_{j}+\lambda C_{j i} \max \left(w_{j}^{*}\left(x^{(\nu)}, y^{(\nu)}\right), 0\right)\right]\right]$

for $i \in \mathcal{D}_{k}$ and

$$
y^{(v+1)}=f\left(\sum_{i \in \mathcal{D}_{k}} s_{i}+\sum_{i \in \mathcal{S}_{k}} \min \left(\frac{\zeta_{i}^{(k)}\left(x^{(v)}, y^{(v)}\right)}{y^{(v)}}, s_{i}\right)\right),
$$

with $\zeta_{i}^{(k)}(x, y)$ for all $i \in \mathcal{S}_{k}$ defined as in Eq. (6). Again, we want to prove that

$$
\left(x^{(v+1)}, y^{(v+1)}\right) \leq\left(x^{(v)}, y^{(v)}\right), \quad v=0,1, \ldots
$$

First, note that for $v=0$,

$$
\begin{aligned}
\sum_{j \in \mathcal{D}_{k}} \Pi_{j i} x_{j}^{(0)}=\sum_{j \in \mathcal{D}_{k}} \Pi_{j i} p_{j}^{(k)} & =\sum_{j \in \mathcal{S}_{k-1} \backslash \mathcal{S}_{k}} \Pi_{j i} p_{j}^{(k)}+\sum_{j \in \mathcal{D}_{k-1}} \Pi_{j i} p_{j}^{(k)} \\
& =\sum_{j \in \mathcal{S}_{k-1} \backslash \mathcal{S}_{k}} \Pi_{j i} \bar{p}_{j}+\sum_{j \in \mathcal{D}_{k-1}} \Pi_{j i} p_{j}^{(k)},
\end{aligned}
$$

observing $\mathcal{D}_{k}=\left(\mathcal{S}_{k-1} \backslash \mathcal{S}_{k}\right) \cup \mathcal{D}_{k-1}$ and $p_{j}^{(k)}=\bar{p}_{j}$ for all $j \in \mathcal{S}_{k-1}$. Second,

$$
\sum_{j \in \mathcal{S}_{k}} C_{j i} \max \left(w_{j}^{*}\left(x^{(0)}, y^{(0)}\right), 0\right)=\sum_{j \in \mathcal{S}_{k-1}} C_{j i} \max \left(w_{j}^{*}\left(p^{(k)}, q^{(k)}\right), 0\right),
$$

since $w_{j}^{*}\left(p^{(k)}, q^{(k)}\right)<0$ for all $j \in \mathcal{S}_{k-1} \backslash \mathcal{S}_{k} \subseteq \mathcal{D}_{k}$. We thus obtain that 


$$
\begin{aligned}
x_{i}^{(1)} & =\alpha\left[r_{i}+s_{i} y^{(0)}\right]+\beta\left[\sum_{j \in \mathcal{D}_{k}} \Pi_{j i} x_{j}^{(0)}+\sum_{j \in \mathcal{S}_{k}}\left[\Pi_{j i} \bar{p}_{j}+\lambda C_{j i} \max \left(w_{j}^{*}\left(x^{(0)}, y^{(0)}\right), 0\right)\right]\right] \\
& =\alpha\left[r_{i}+s_{i} q^{(k)}\right]+\beta\left[\sum_{j \in \mathcal{D}_{k-1}} \Pi_{j i} p_{j}^{(k)}+\sum_{j \in \mathcal{S}_{k-1}}\left[\Pi_{j i} \bar{p}_{j}+\lambda C_{j i} \max \left(w_{j}^{*}\left(p^{(k)}, q^{(k)}\right), 0\right)\right]\right] .
\end{aligned}
$$

For $i \in \mathcal{D}_{k-1}$ this shows that $x_{i}^{(1)}=p_{i}^{(k)}=x_{i}^{(0)}$. For the remaining case $i \in \mathcal{D}_{k} \backslash \mathcal{D}_{k-1}$, we have

$$
\begin{aligned}
x_{i}^{(1)} & \leq r_{i}+s_{i} q^{(k)}+\sum_{j \in \mathcal{D}_{k-1}} \Pi_{j i} p_{j}^{(k)}+\sum_{j \in \mathcal{S}_{k-1}}\left[\Pi_{j i} \bar{p}_{j}+\lambda C_{j i} \max \left(w_{j}^{*}\left(p^{(k)}, q^{(k)}\right), 0\right)\right] \\
& =r_{i}+s_{i} q^{(k)}+\sum_{j=1}^{n} \Pi_{j i} p_{j}^{(k)}+\lambda \sum_{j=1}^{n} C_{j i} \max \left(w_{j}^{*}\left(p^{(k)}, q^{(k)}\right), 0\right)<\bar{p}_{i}=p_{i}^{(k)}=x_{i}^{(0)} .
\end{aligned}
$$

Here, the first inequality holds because $0 \leq \alpha, \beta \leq 1$. The first stated equality follows from $p_{j}^{(k)}=\bar{p}_{j}$ for $j \in \mathcal{S}_{k-1}$ and the fact that $w_{j}^{*}\left(p^{(k)}, q^{(k)}\right)<0$ for all $j \in \mathcal{D}_{k-1} \subseteq \mathcal{D}_{k}$. The last inequality results from $i \in \mathcal{D}_{k} \backslash \mathcal{D}_{k-1} \subseteq \mathcal{S}_{k-1}$.

For the price of the illiquid asset, we first observe that for all $i \in \mathcal{S}_{k}$, $\zeta_{i}^{(k)}\left(x^{(0)}, y^{(0)}\right)=\zeta_{i}^{(k-1)}\left(p^{(k)}, q^{(k)}\right)$ by using the same arguments as for Eq. (13). From this it follows that

$$
\begin{aligned}
y^{(1)} & =f\left(\sum_{i \in \mathcal{D}_{k-1}} s_{i}+\sum_{i \in \mathcal{S}_{k-1} \backslash \mathcal{S}_{k}} s_{i}+\sum_{i \in \mathcal{S}_{k}} \min \left(\frac{\zeta_{i}^{(k-1)}\left(p^{(k)}, q^{(k)}\right)}{q^{(k)}}, s_{i}\right)\right) \\
& \leq f\left(\sum_{i \in \mathcal{D}_{k-1}} s_{i}+\sum_{i \in \mathcal{S}_{k-1}} \min \left(\frac{\zeta_{i}^{(k-1)}\left(p^{(k)}, q^{(k)}\right)}{q^{(k)}}, s_{i}\right)\right)=q^{(k)}=y^{(0)},
\end{aligned}
$$

because $f$ is monotonically decreasing and the last step follows from the definition of $q^{(k)}$. This proves (12) for $v=0$; the arguments for $v>0$ are analogous. This implies that $\left(p^{(k+1)}, q^{(k+1)}\right)=\lim _{v}\left(x^{(v)}, y^{(v)}\right) \leq$ $\left(x^{(0)}, y^{(0)}\right)=\left(p^{(k)}, q^{(k)}\right)$. This finishes the induction step $k \rightarrow k+1$, and thus completes the first step of the proof, i.e., the sequence of pricepayment pairs $\left(p^{(k)}, q^{(k)}\right)$ decreases.

(ii) In the second step, we need to show that $\left(p^{(k)}, q^{(k)}\right) \geq\left(p^{+}, q^{+}\right)$for all $k=0,1, \ldots, n$. Again, this will be established by induction. For $k=0$ the assertion is obvious, since $\left(p^{(0)}, q^{(0)}\right)=\left(\bar{p}, q_{0}\right) \geq\left(p^{+}, q^{+}\right)$by Lemma 3.1 (a).

For the induction step $k \rightarrow k+1$, we observe that by the induction hypothesis a bank that defaults under $\left(p^{(k)}, q^{(k)}\right)$ does also default under $\left(p^{+}, q^{+}\right)$, thus $\mathcal{D}\left(p^{+}, q^{+}\right) \supseteq \mathcal{D}_{k}$. Now, for $i \in \mathcal{S}_{k}$, one has $p_{i}^{(k+1)}=\bar{p}_{i} \geq p_{i}^{+}$. It again remains to analyze the entries of $p^{(k+1)}$ belonging to banks in $\mathcal{D}_{k}$. Therefore, we reuse the recursive principle stated in Eq. (10) together with (11), and prove that for all $v=0,1, \ldots: x_{i}^{(v)} \geq p_{i}^{+}\left(i \in \mathcal{D}_{k}\right), y^{(v)} \geq q^{+}$. Now, starting again 
with $x_{i}^{(0)}=p_{i}^{(k)}$ for $i \in \mathcal{D}_{k}$ and $y^{(0)}=q^{(k)}$, we obtain for $i \in \mathcal{D}_{k}$ that

$$
\begin{aligned}
x_{i}^{(1)} & =\alpha\left[r_{i}+s_{i} q^{(k)}\right]+\beta\left[\sum_{j \in \mathcal{D}_{k}} \Pi_{j i} p_{j}^{(k)}+\sum_{j \in \mathcal{S}_{k}}\left[\Pi_{j i} \bar{p}_{j}+\lambda C_{j i} \max \left(w_{j}^{*}\left(p^{(k)}, q^{(k)}\right), 0\right)\right]\right] \\
& \geq \alpha\left[r_{i}+s_{i} q^{+}\right]+\beta\left[\sum_{j \in \mathcal{D}_{k}} \Pi_{j i} p_{j}^{+}+\sum_{j \in \mathcal{S}_{k}}\left[\Pi_{j i} \bar{p}_{j}+\lambda C_{j i} \max \left(w_{j}^{*}\left(p^{+}, q^{+}\right), 0\right)\right]\right] \\
& \geq \alpha\left[r_{i}+s_{i} q^{+}\right]+\beta\left[\sum_{j=1}^{n} \Pi_{j i} p_{j}^{+}+\lambda \sum_{j=1}^{n} C_{j i} \max \left(w_{j}^{*}\left(p^{+}, q^{+}\right), 0\right)\right]=p_{i}^{+} .
\end{aligned}
$$

Here, the first inequality holds because of the induction hypothesis and Lemma 2.2 (b). The second inequality follows from $\bar{p} \geq p^{+}$and $w_{j}^{*}\left(p^{+}, q^{+}\right)<0$ for all $j \in \mathcal{D}_{k} \subseteq \mathcal{D}\left(p^{+}, q^{+}\right)$. Finally, $\left(p^{+}, q^{+}\right)$is a price-payment equilibrium by assumption; thus, the last equality holds for $i \in \mathcal{D}_{k} \subseteq \mathcal{D}\left(p^{+}, q^{+}\right)$according to Definition 3.1.

Regarding the price of the illiquid asset, we first obtain by the induction hypothesis that

$$
\begin{aligned}
\zeta_{i}^{(k)}\left(p^{(k)}, q^{(k)}\right) \leq \zeta_{i}^{(k)}\left(p^{+}, q^{+}\right), \text {for all } i \in \mathcal{S}_{k} \text {. This leads to } \\
y^{(1)}=f\left(\sum_{i \in \mathcal{D}_{k}} s_{i}+\sum_{i \in \mathcal{S}_{k}} \min \left(\frac{\zeta_{i}^{(k)}\left(p^{(k)}, q^{(k)}\right)}{\left.\left.q^{(k)}, s_{i}\right)\right)}\right.\right. \\
\quad \geq f\left(\sum_{i \in \mathcal{D}\left(p^{+}, q^{+}\right)} s_{i}+\sum_{i \in \mathcal{S}\left(p^{+}, q^{+}\right)} \min \left(\frac{\zeta_{i}^{(k)}\left(p^{+}, q^{+}\right)}{q^{+}}, s_{i}\right)\right)=q^{+},
\end{aligned}
$$

using similar arguments as before. Hence, by the recursive definition of the sequence $\left(x^{(v)}, y^{(v)}\right)$, we see that every element of this sequence will be larger than or equal to the corresponding entries of the greatest price-payment equilibrium. Overall, this yields $\left(p^{(k)}, q^{(k)}\right) \geq\left(p^{+}, q^{+}\right)$for all $k=0,1, \ldots, n$, as desired.

(iii) To finish this proof, we combine all previous arguments. By our first step, the payment vectors are decreasing with each iteration of the algorithm and hence, $\mathcal{D}_{k+1} \supseteq \mathcal{D}_{k}$, which leads to two possible cases. The first is $\mathcal{D}_{k+1}=\mathcal{D}_{k}$. In this case, the algorithm terminates and due to the fixed-point construction, $\left(p^{(k)}, q^{(k)}\right)$ is a price-payment equilibrium as in Definition 3.1. Moreover, as $\left(p^{+}, q^{+}\right)$is the unique greatest price-payment equilibrium and by our second step $\left(p^{(k)}, q^{(k)}\right) \geq\left(p^{+}, q^{+}\right)$, the algorithm terminates with the greatest pricepayment equilibrium. The second possibility for the sequence of default sets is that it is strictly increasing from one round to another, i.e., $\mathcal{D}_{k+1} \supset \mathcal{D}_{k}$. This means that a new bank joined the default set, and payments and prices have to be adjusted. But since there are at most $n$ banks that can join the default set, after at most $n+1$ iterations $^{11}$ the default set does not change anymore.

\footnotetext{
${ }^{11}$ In the first round, $k=0$, the default set can be empty as the contagion process may solely be triggered by the asset price effect.
} 
Thus, we eventually end up in the first possible case, finding the greatest pricepayment equilibrium.

\section{Appendix 1. Example: price-payment equilibria}

Price-payment equilibria are non-unique and, moreover, the set of equilibria is not necessarily connected. In the following example, we will construct a financial system in which for some $p \in \mathbb{R}^{n}$ with $p^{-}<p<p^{+}$there does not exist a $q \in\left[q^{-}, q^{+}\right]$ such that $(p, q)$ is a price-payment equilibrium. We will also show that for given $q \in \mathbb{R}$ with $q^{-}<q<q^{+}$it is not always possible to find $p \in\left[p^{-}, p^{+}\right]$such that $(p, q)$ is a price-payment equilibrium.

For the analysis of the counterexample, we recall two necessary conditions for price-payment equilibria $\left(p^{*}, q^{*}\right)$ from Definition 2.3:

1. For a given clearing price $q^{*}$, the clearing payment vector $p^{*}$ satisfies the relation

$$
p^{*}=\chi\left(p^{*}, q^{*}\right) \text {. }
$$

For any fixed $q \in\left[q_{\min }, q_{0}\right]$, we call a corresponding fixed point of Eq. (14) a clearing vector for $q$, denoted by $p^{* q}$. Its existence is established by Tarski's fixed-point theorem, analogous to the proof of Theorem 3.2.

2. Analogously, for fixed $p \in[\mathbf{0}, \bar{p}]$, we define a clearing price for $p$ by

$$
q^{* p}=f\left(\theta\left(p, q^{* p}\right)\right) .
$$

Its existence follows again from Tarski's fixed-point theorem.

Consider the following financial system:

$$
\Pi=\left(\begin{array}{ll}
0 & 0.4 \\
0.4 & 0
\end{array}\right), r=\left(\begin{array}{l}
0.5 \\
0.5
\end{array}\right), s=\left(\begin{array}{l}
1 \\
2
\end{array}\right), \bar{p}=\left(\begin{array}{l}
1 \\
1
\end{array}\right),
$$

$\alpha=\beta=0.5$, with cross-holdings $C$ set to zero, and the inverse demand function $f(x)=\exp (-x)$. We obtain the greatest and least price-payment equilibrium by Algorithm 3.4 and Remark 3.6:

$$
\left(p^{-}, q^{-}\right) \approx\left(\left(\begin{array}{l}
0.3488 \\
0.3695
\end{array}\right), 0.0498\right), \quad\left(p^{+}, q^{+}\right) \approx\left(\left(\begin{array}{l}
1 \\
1
\end{array}\right), 0.7717\right) .
$$

We first demonstrate that there is no price-payment equilibrium $(p, q)$ with $p=$ $(0.4,0.5)^{T}$, although $p^{-}<p<p^{+}$. If $p=(0.4,0.5)^{T}$ was a clearing vector, then by (14)

$$
p_{i}=\chi_{i}(p, q)=\alpha\left(r_{i}+s_{i} q\right)+\beta\left(\sum_{j \in \mathcal{N}} \Pi_{j i} p_{j}\right), \quad i=1,2 .
$$

This follows since both banks are in default and faced with bankruptcy costs. However, there is no $q$ solving both equations simultaneously. Hence, there is no $q \in$ $\left[q^{-}, q^{+}\right]$such that $p=(0.4,0.5)^{T}$ becomes a clearing vector for $q$. 
Second, we demonstrate that there is no price-payment equilibrium $(p, q)$ with $q=0.05$, although $q^{-}<q<q^{+}$. If $q$ was a clearing price, then by (15):

$$
0.05=q=f(\theta(p, 0.05))=\exp (-\theta(p, 0.05))=\exp (-3)=0.0498 \text {, }
$$

since $\theta(p, 0.05)=3$ for all $p \in\left[p^{-}, p^{+}\right]$. This is a contradiction.

\section{Appendix 2. Cross-holdings with price impact}

As explained in the section "An integrated financial network model", we assume that the liquidation of cross-holdings is subject to price impact. So far, this was simply encoded by the parameter $\lambda \in \mathbb{R}^{n}$ which referred to a fixed fraction that can be realized. A more sophisticated approach consists in specifying an inverse demand function for cross-holdings. Letting $q^{C} \in \mathbb{R}^{n}$ be the vector of net worth prices for the cross-holdings in the $n$ banks and $\theta^{C}\left[w, q^{C}\right] \in \mathbb{R}^{n}$ the amount of cross-holdings liquidated for a net worth vector $w \in \mathbb{R}^{n}$, the price vector $q^{C}$ is given as a fixed point of the inverse demand function $f^{C}: \mathbb{R}^{n} \rightarrow[0,1]^{n}=[\mathbf{0}, \mathbf{1}] \subset \mathbb{R}^{n}$, i.e.,

$$
q^{C}=f^{C}\left(\theta^{C}\left[w, q^{C}\right]\right),
$$

with $f^{C}(\mathbf{0})=\mathbf{1}$ and $x \mapsto f^{C}(x)$ monotonically decreasing. In case of liquidation, the value of bank $i$ 's cross-holdings is given by $\sum_{j \in \mathcal{N}} C_{j i} \max \left(w_{j}, 0\right) q_{j}^{C}$.

As before, we suppose that banks liquidate their cross-holdings proportionally; i.e., each bank $j \in \mathcal{N}$ calculates the proportion of cross-holdings it needs to liquidate:

$$
v_{j}^{C}\left[w, q^{C}\right]=\min \left(\frac{\max \left[\bar{p}_{j}-r_{j}-\sum_{k \in \mathcal{N}} \Pi_{k j} p_{k}-\left(1-\mathbb{I}_{j}\right) s_{j} q, 0\right]}{\sum_{k \in \mathcal{N}} C_{k j} \max \left(w_{k}, 0\right) q_{k}^{C}}, 1\right) ;
$$

thus, the total quantity of bank $i$ 's shares that is liquidated by other banks equals

$$
\theta_{i}^{C}\left[w, q^{C}\right]=\sum_{j \in \mathcal{N}} C_{i j} v_{j}^{C}\left[w, q^{C}\right] .
$$

The net worth price vector $q^{C}$ depends on the banks' net worths vector $w$ and vice versa. We characterize these values as a combined equilibrium:

Definition B.1 For fixed payments $p \in \mathbb{R}_{+}^{n}$ and a price of the illiquid asset $q \in$ $\mathbb{R}_{+}$, a net worth equilibrium $\left[w^{*}, q^{C *}\right] \in \mathbb{R}^{n} \times \mathbb{R}^{n}$ is a fixed point of the function $\Psi^{C}: \mathbb{R}^{n} \times \mathbb{R}^{n} \rightarrow \mathbb{R}^{n} \times \mathbb{R}^{n}$ defined by:

$$
\begin{aligned}
\Psi^{C}\left[w, q^{C}\right]= & \left\{r+s q+\Pi^{T} p+\operatorname{diag}\left(v^{C}\right) C^{T} \operatorname{diag}\left(q^{C}\right)(w \vee \mathbf{0})+\left(I-\operatorname{diag}\left(v^{C}\right)\right) C^{T}(w \vee \mathbf{0})\right\} \\
& \times\left\{f^{C}\left(\theta^{C}\left[w, q^{C}\right]\right)\right\} .
\end{aligned}
$$

The existence of a greatest and least net worth equilibrium follows again from Tarski's fixed-point theorem, now applied to the function $\Psi^{C}$. 
Note that the modeling framework that we consider in the numerical case studies above is a special case of the general inverse demand function. Setting $\lambda_{i}=(1-c) \kappa$ with $0 \leq \kappa \leq 1$, the corresponding inverse demand function $f^{C}$ is defined by its components $f_{j}^{C}(x)=(1-c) \kappa, j \in \mathcal{N}$.

\section{Authors' contributions}

Both authors jointly conducted the research and wrote the paper. Both authors read and approved the final manuscript.

\section{Competing interests}

The authors declare that they have no competing interests.

\section{References}

Amini, H, Filipović, D, Minca, A: Systemic risk with central counterparty clearing. Swiss Finance Institute Research Paper No. 13-34 (2013)

Amini, H, Filipović, D, Minca, A: Uniqueness of equilibrium in a payment system with liquidation costs. Oper. Res. Lett 44(1), 1-5 (2016)

Boucinha, M, Holton, S, Tiseno, A: Bank equity valuations and credit supply. Working Paper (2017). https://www.oenb.at/dam/jcr:c49e6115-3592-45fd-8416-6ed37332e201/4_paper_bank_equity_ valuations_and_credit_supply_boucinha.pdf

Brioschi, F, Buzzacchi, L, Colombo, MG: Risk capital financing and the separation of ownership and control in business groups. J. Bank. Finance 13(4), 747-772 (1989)

Brunnermeier, MK: Deciphering the liquidity and credit crunch 2007-2008. J. Econ. Perspect 23(1), 77-100 (2009)

Chen, N, Liu, X, Yao, DD: An optimization view of financial systemic risk modeling: Network effect and market liquidity effect. Oper. Res 64(5), 1089-1108 (2016)

Cifuentes, R, Ferrucci, G, Shin, HS: Liquidity risk and contagion. J. Eur. Econ. Assoc 3(2-3), 556-566 (2005)

Cont, R, Wagalath, L: Fire sales forensics: Measuring endogenous risk. Math. Finance 26(4), 835-866 (2016)

Cont, R, Moussa, A, Santos, EB: Network structure and systemic risk in banking systems. Handbook on Systemic Risk, pp. 327-368. Cambridge University Press (2013)

Coval, J, Stafford, E: Asset fire sales (and purchases) in equity markets. J. Financial Econ 86(2), 479-512 (2007)

Craig, B, von Peter, G: Interbank tiering and money center banks. J. Financial Intermediation 23(3), 322-347 (2014)

Drehmann, M, Tarashev, N: Measuring the systemic importance of interconnected banks. BIS Working Paper 342, Bank for International Settlements (2011)

Eisenberg, L, Noe, TH: Systemic risk in financial systems. Manag. Sci 47(7), 236-249 (2001)

Elliott, M, Golub, B, Jackson, MO: Financial networks and contagion. Am. Econ. Rev 104(10), 3115-3153 (2014)

Elsinger, H: Financial networks, cross holdings, and limited liability. Working Papers 156, Österreichische Nationalbank (Austrian Central Bank) (2009)

Elsinger, H, Lehar, A, Summer, M: Risk assessment for banking systems. Manage. Sci 52(9), 1301-1314 (2006)

Erdös, P, Rényi, A: On random graphs, I. Publicationes Mathematicae (Debrecen) 6, 290-297 ((1959))

Fedenia, M, Hodder, JE, Triantis, AJ: Cross-holdings: Estimation issues, biases, and distortions. Rev. Financial Stud 7(1), 61-96 (1994)

Feinstein, Z: Financial contagion and asset liquidation strategies. Oper. Res. Lett 45(2), 109-114 (2017) 
Feinstein, Z, Rudloff, B, Weber, S: Measures of systemic risk. SIAM J. Financial Math (2017)

Fischer, T: No-arbitrage pricing under systemic risk: Accounting for cross-ownership. Math. Finance 24(1), 97-124 (2014)

Föllmer, H, Schied, A Stochastic Finance - An Introduction in Discrete Time, 3rd edn. De Gruyter, Berlin (2011)

Föllmer, H, Weber, S: The Axiomatic Approach to Risk Measurement for Capital Determination. Ann. Rev. Financial Econ 7, 301-337 (2015)

Gai, P, Kapadia, S: Contagion in financial networks. Bank of England Working Papers 383, Bank of England (2010)

Glasserman, P, Young, HP: How likely is contagion in financial networks? J. Bank. Finance 50, 383-399 (2015)

Jotikasthira, C, Lundblad, C, Ramadorai, T: Asset fire sales and purchases and the international transmission of funding shocks. J. Finance 67(6), 2015-2050 (2012)

Karl, S, Fischer, T: Cross-ownership as a structural explanation for over- and underestimation of default probability. Quant Finance 14(6), 1031-1046 (2014)

Khandani, AE, Lo, AW: What happened to the quants in August 2007? J. Financial Markets 14(1), 1-46 (2011)

Nier, E, Yang, J, Yorulmazer, T, Alentorn, A: Network models and financial stability. J. Econ. Dynam. Control 31(6), 2033-2060 (2007)

Rogers, LCG, Veraart, LAM: Failure and rescue in an interbank network. Manag. Sci 59(4), 882-898 (2013)

Shleifer, A, Vishny, RW: Liquidation values and debt capacity: A market equilibrium approach. J. Finance 47(4), 1343-1366 (1992)

Shleifer, A, Vishny, RW: Fire sales in finance and macroeconomics. J. Econ. Perspect 25(1), 29-48 (2011)

Staum, J: Counterparty contagion in context: Contributions to systemic risk. Handbook on Systemic Risk, pp. 512-548. Cambridge University Press (2013)

Suzuki, T: Valuing corporate debt : The effect of cross-holdings of stock and debt. J. Oper. Res. Soc. Japan 45(2), 123-144 (2002)

Tarski, A: A lattice-theoretical fixpoint theorem and its applications. Pac. J. Math 5(2), 285-309 (1955)

Upper, C: Using counterfactual simulations to assess the danger of contagion in interbank markets. BIS Working Papers 234, Bank for International Settlements (2007)

Upper, C: Simulation methods to assess the danger of contagion in interbank markets. J. Financial Stab 7(3), 111-125 (2011)

van Lelyfeld, I, in't Veld, D: Finding the core: Network structure in interbank markets. J. Bank. Finance 49, 27-40 (2014) 